\title{
Article \\ Organocatalytic Asymmetric [2 + 4] Cycloadditions of 3-Vinylindoles with ortho-Quinone Methides
}

\author{
Si-Jia Liu ${ }^{1}$, Man-Su Tu ${ }^{1}$, Kai-Yue Liu ${ }^{1}$, Jia-Yi Chen ${ }^{2}$, Shao-Fei Ni ${ }^{2, *}$, Yu-Chen Zhang ${ }^{1, *}$ and Feng Shi ${ }^{1, *}$ \\ 1 School of Chemistry and Materials Science, Jiangsu Normal University, Xuzhou 221116, China; \\ yoghourt1128@163.com (S.J.L.); manmandexiaowu1987@163.com (M.-S.T.); L15383434496@163.com (K.-Y.L.) \\ 2 Department of Chemistry, Key Laboratory for Preparation and Application of Ordered Structural Materials of \\ Guangdong Province, Shantou University, Shantou 515063, China; 19jychen4@stu.edu.cn \\ * Correspondence: sfni@stu.edu.cn (S.-F.N.); zhangyc@jsnu.edu.cn (Y.-C.Z.); fshi@jsnu.edu.cn (F.S.)
}

check for

updates

Citation: Liu, S.-J.; Tu, M.-S.; Liu, K.-Y.; Chen, J.-Y.; Ni, S.-F.; Zhang, Y.-C.; Shi, F. Organocatalytic Asymmetric [2+4] Cycloadditions of 3-Vinylindoles with ortho-Quinone Methides. Molecules 2021, 26, 6751. https://doi.org/10.3390/

molecules 26216751

Academic Editor: Ming-Hua Xu

Received: 3 August 2021

Accepted: 6 November 2021

Published: 8 November 2021

Publisher's Note: MDPI stays neutral with regard to jurisdictional claims in published maps and institutional affiliations.

Copyright: (c) 2021 by the authors. Licensee MDPI, Basel, Switzerland. This article is an open access article distributed under the terms and conditions of the Creative Commons Attribution (CC BY) license (https:/ / creativecommons.org/licenses/by/ $4.0 /)$.

\begin{abstract}
Catalytic asymmetric [2 + 4] cycloadditions of 3-vinylindoles with ortho-quinone methides and their precursors were carried out in the presence of chiral phosphoric acid to afford a series of indole-containing chroman derivatives with structural diversity in overall high yields (up to 98\%), good diastereoselectivities (up to $93: 7 \mathrm{dr}$ ) and moderate to excellent enantioselectivities (up to $98 \%$ ee). This approach not only enriches the chemistry of catalytic asymmetric cycloadditions involving 3-vinylindoles but is also useful for synthesizing chiral chroman derivatives.
\end{abstract}

Keywords: vinylindoles; cycloaddition; ortho-quinone methide; chiral phosphoric acid; asymmetric organocatalysis

\section{Introduction}

Chiral indole derivatives are ubiquitous in biologically important natural products, pharmaceuticals and materials [1-5]. In recent years, vinylindoles have been recognized as versatile reactants for the synthesis of enantioenriched indole derivatives [6,7]. The 3-vinylindoles belong to a class of vinylindoles with multiple reactive sites and are widely applied in organocatalytic asymmetric cycloadditions and substitutions [8-29].

As shown in Figure 1, 3-vinylindoles exhibit versatile reactivities and participate in four main types of organocatalytic asymmetric reactions. Namely, 3-vinylindoles act as dienes in asymmetric [4 + 2] cycloaddition (Figure 1a) [8-12], as mono-olefins in asymmetric [2 $+n]$ cycloaddition (Figure 1b) [13-23], as electrophiles in asymmetric addition reaction (Figure 1c) [24-27], and as nucleophiles in asymmetric alkenylation (Figure 1d) [28,29]. Among these reactions, organocatalytic asymmetric $[2+n]$ cycloaddition of 3-vinylindoles as mono-olefins has proven to be an important reaction (Figure 1b) to efficiently synthesize indole-containing heterocycles with optical purity [30,31]. (a)

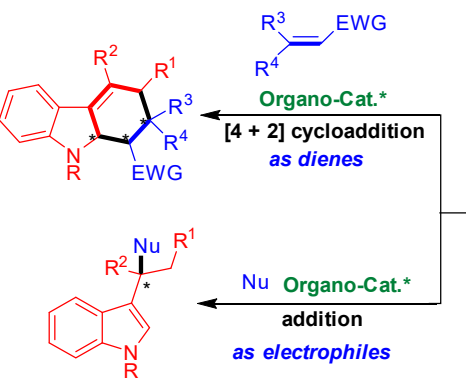

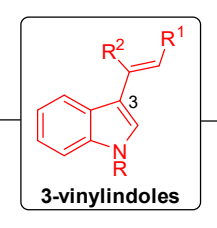

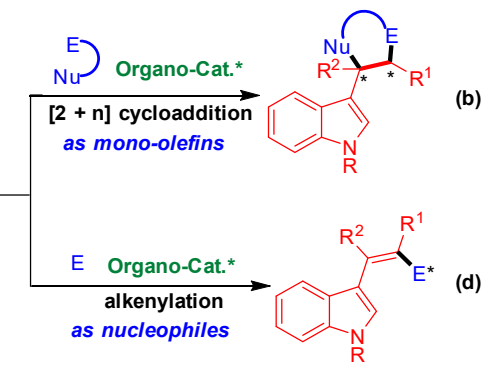

Figure 1. Profile of organocatalytic asymmetric reactions involving 3-vinylindoles. (a) $[4+2]$ Cycloaddition; (b) [2 + n] Cycloaddition; (c) Addition reaction; (d) Alkenylation reaction. The asterisk * indicates chiral center. 
Among the organocatalytic asymmetric [2+n] cycloadditions of 3-vinylindoles, [2+4] cycloadditions using 3-vinylindoles as dienophiles belong to a class of important inverseelectron-demand Diels-Alder reactions (Figure 2). However, most of these reactions involve [2 +4] cycloadditions of 3-vinylindoles with aza-dienes (Figure 2a) [13-15]. By sharp contrast, organocatalytic asymmetric [2+4] cycloadditions of 3-vinylindoles with oxa-dienes have been sporadically reported in the literature (Figure 2b) [16-18]. The underdevelopment of this class of reactions could be ascribed to the considerable challenges encountered in conducting these reactions, which mainly include (1) finding suitable reaction partners and (2) controlling the regioselectivity (whether 3-vinylindoles act as mono-olefins or dienes) and enantioselectivity of the cycloaddition reaction.

(a) [2+4] cycloadditions of 3-vinylindoles with aza-dienes: Well-developed

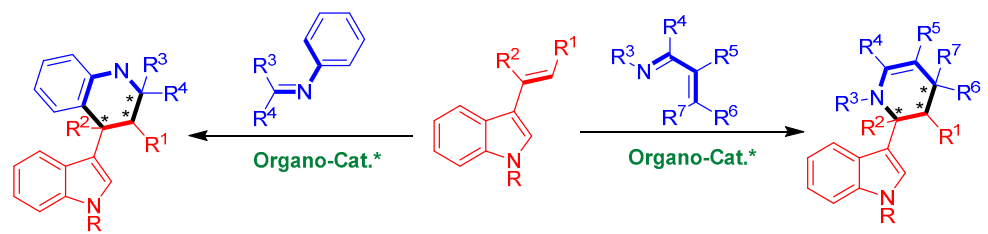

(b) $[2+4]$ cycloadditions of 3-vinylindoles with oxa-dienes: Less developed

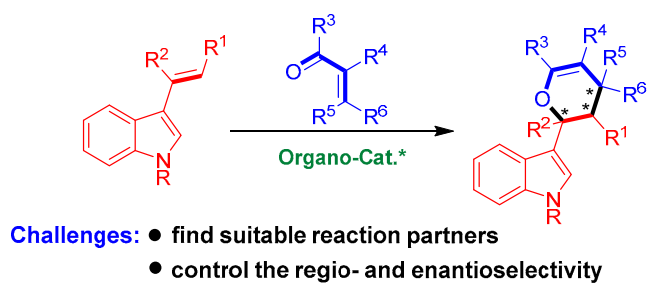

Figure 2. Profile of organocatalytic asymmetric [2+4] cycloadditions of 3-vinylindoles. The asterisk * indicates chiral center.

To date, there are only three cases of organocatalytic asymmetric [2+4] cycloadditions of 3-vinylindoles with oxa-dienes (Figure 3) [16-18]. In 2011, Zhu's group realized the organocatalytic asymmetric [2+4] cycloaddition of 3-vinylindoles with chromone-derived oxa-dienes, generating enantioenriched indole-containing heterocycles (Figure 3a) [16]. In 2019, Zhang and coworkers performed an organocatalytic asymmetric [2+4] cycloaddition of 3-vinylindoles with $\beta, \gamma$-unsaturated $\alpha$-ketoesters with high enantioselectivities (Figure $3 b$ ) [17]. Very recently, our group reported an organocatalytic asymmetric [2+4] cycloaddition of 3-vinylindoles with ortho-hydroxyphenyl-substituted para-quinone methide derivatives that provides a series of chiral chroman derivatives bearing an indole moiety (Figure 3c) [18]. Other than these cases, organocatalytic asymmetric [2 +4$]$ cycloadditions of 3-vinylindoles with oxa-dienes remain rather limited. Therefore, developing this class of reactions and overcoming the associated inherent challenges are urgently required.

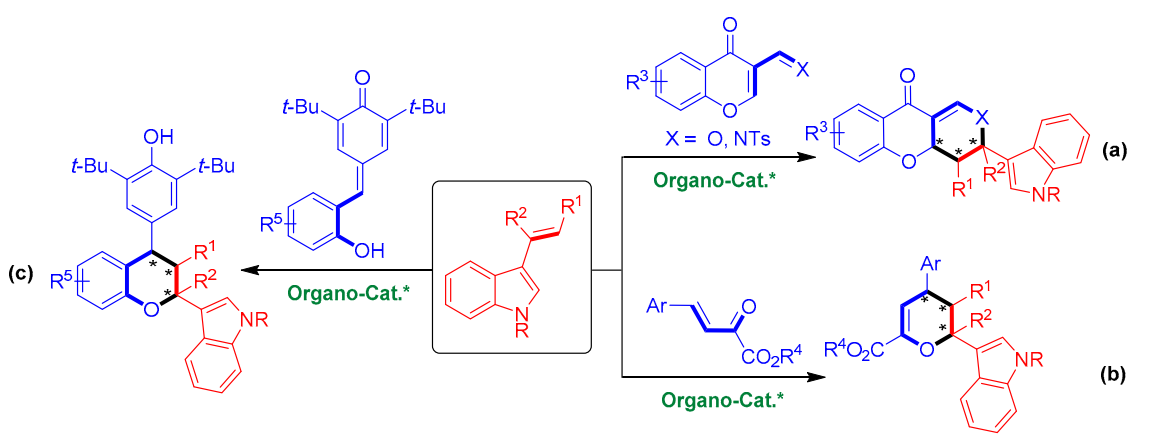

Figure 3. Limited examples of organocatalytic asymmetric $[2+4]$ cycloadditions of 3-vinylindoles with oxa-dienes. (a) With chromone-derived oxa-dienes; (b) With $\beta, \gamma$-unsaturated $\alpha$-ketoesters; (c) With ortho-hydroxyphenyl-substituted para-quinone methides. The asterisk* indicates chiral center. 
To fulfill this task and in continuation of our ongoing efforts in the enantioselective synthesis of indole-based chiral heterocycles [32-35], we designed a chiral phosphoric acid [36-44] (CPA)-catalyzed asymmetric [2 + 4] cycloaddition of 3-vinylindoles with ortho-quinone methides (o-QMs) and their precursors (Figure 4 ). The $o$-QMs were selected as suitable reaction partners because of their high reactivity as oxa-dienes in catalytic asymmetric cycloadditions [45-63]. Within this design scheme, 3-vinylindoles $\mathbf{1}$ and $o$-QMs 2 can be simultaneously activated by CPA via hydrogen-bonding interactions. This dual activation mode of CPA facilitates regioselective and enantioselective [2+4] cycloaddition between 3-vinylindoles 1 and o-QMs 2, thus affording the chiral indole-containing chroman derivatives 3 .

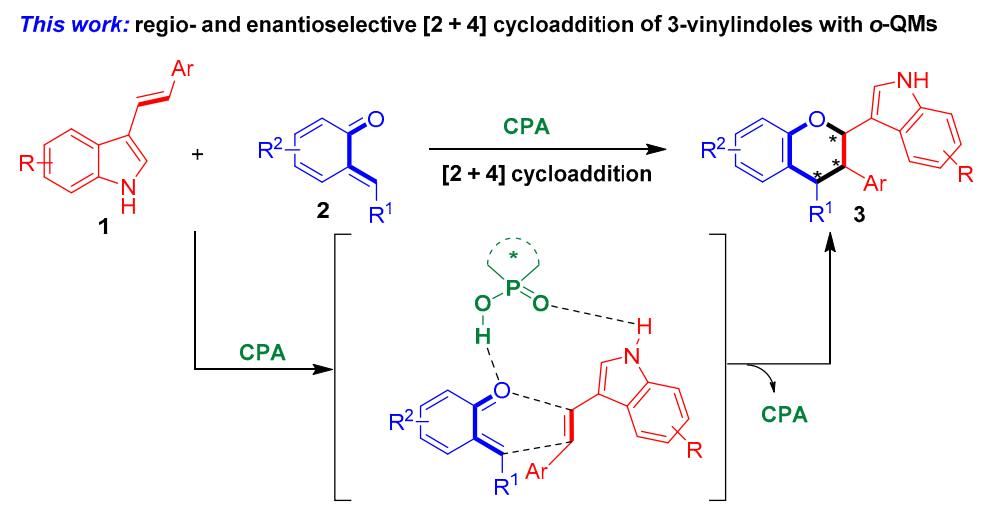

Figure 4. Design of CPA-catalyzed asymmetric [2 +4] cycloaddition of 3-vinylindoles using o-QMs. The asterisk * indicates chiral center.

Herein, we report the CPA-catalyzed asymmetric [2 + 4] cycloaddition of 3-vinylindoles with $o$-QMs and their precursors to afford chiral indole-containing chroman derivatives in overall good yields (up to $98 \%$ yield) and moderate to excellent stereoselectivities (up to 93:7 dr, 98\% ee).

\section{Results and Discussion}

2.1. Organocatalytic Asymmetric [2 + 4] Cycloaddition of 3-Vinylindoles with Sesamol-Derived $o-Q M s$

\subsubsection{Optimization of Reaction Conditions}

To test the feasibility of the designed catalytic asymmetric $[2+4]$ cycloaddition, 3-vinylindole 1a was reacted with sesamol-derived o-QM $\mathbf{2 a}$ in the presence of CPA $(\boldsymbol{R})$ $4 \mathbf{a}$ at $25{ }^{\circ} \mathrm{C}$ in toluene (Table 1 , entry 1 ). Catalytic asymmetric [ +4$]$ cycloaddition occurred, as expected, to afford the chiral chroman derivative 3aa in a moderate yield with excellent diastereoselectivity, albeit with no enantio-control (43\% yield, 96:4 dr, 0\% ee). To control the enantioselectivity of this [2+4] cycloaddition, a series of CPAs 4 were screened (entries 2-7). The CPA (R)-4c bearing two bulky 3,3'-(1-naphthyl) groups facilitated the $[2+4]$ cycloaddition with the highest enantioselectivity of $66 \%$ ee (for the major diastereomer) among the investigated catalysts (entry 3 vs. entries 1-2 and 4-7), which could be ascribed to the steric hindrance effect of the $3,3^{\prime}$-disubstituents of CPA in creating a chiral environment for controlling the enantioselectivity $[64,65]$. The subsequent evaluation of a series of representative solvents (entries 8-12) in the presence of CPA (R)-4c showed that toluene remained the most suitable solvent in terms of controlling the enantioselectivity (entry 3 vs. entries 8-12).

Next, we investigated the effect of the reaction temperature (Table 2, entries 1-4) and found $0{ }^{\circ} \mathrm{C}$ to be the optimal reaction temperature (entry 1 vs. entry 3 ). Modulating the molar ratio of the reactants (entries 5-8) revealed that increasing the quantity of sesamolderived o-QM 2a improved the yield but decreased the enantioselectivity (entry 3 vs. entries 5-6), whereas increasing the quantity of 3-vinylindole 1a was detrimental to the reaction (entry 3 vs. entries 7-8). Therefore, the most suitable molar reagent ratio remained 1:1.2. 
Finally, some additives were screened (entries 9-13), and the optimal conditions for this $[2+4]$ cycloaddition were set as shown in entry 12 .

Table 1. Screening of catalysts and solvents ${ }^{a}$.

\begin{tabular}{|c|c|c|c|c|c|}
\hline & & $(R)-4$ & $\begin{array}{l}\mathbf{4 a}, \mathrm{G}=4-\mathrm{CIC}_{6} \mathrm{H}_{4} \\
\mathbf{4 b}, \mathrm{G}=2 \text {-naphthyl } \\
\mathbf{4 c}, \mathrm{G}=1 \text {-naphthyl } \\
\mathbf{4 d}, \mathrm{G}=9 \text {-phenanthrenyl } \\
\mathbf{4 e ,}, \mathrm{G}=9 \text {-anthracenyl } \\
\mathbf{4 f}, \mathrm{G}=2,4,6-(i-\mathrm{Pr})_{3} \mathrm{C}_{6} \mathrm{H}_{2} \\
\mathbf{4 g}, \mathrm{G}=\mathrm{SiPh}_{3}\end{array}$ & & \\
\hline & & $\widehat{P}_{\mathrm{Ph}}$ & $\underset{\text { ent, } 25^{\circ} \mathrm{C}, 12 \mathrm{~h}}{\longrightarrow}$ & 3aa & \\
\hline Entry & 4 & Solvent & Yield (\%) ${ }^{b}$ & $\mathrm{Dr}^{c}$ & $\operatorname{Ee}(\%)^{d}$ \\
\hline 1 & $4 a$ & toluene & 43 & $96: 4$ & 0 \\
\hline 2 & $4 b$ & toluene & 44 & $97: 3$ & 5 \\
\hline 3 & $4 c$ & toluene & 67 & $82: 18$ & 66 \\
\hline 4 & $4 d$ & toluene & 55 & $90: 10$ & 0 \\
\hline 5 & $4 e$ & toluene & 61 & $84: 16$ & 51 \\
\hline 6 & $4 \mathrm{f}$ & toluene & 32 & $88: 12$ & 12 \\
\hline 7 & $4 g$ & toluene & 44 & $96: 4$ & 4 \\
\hline 8 & $4 c$ & $\mathrm{ClCH}_{2} \mathrm{CH}_{2} \mathrm{Cl}$ & 89 & $86: 14$ & 62 \\
\hline 9 & $4 c$ & 1,4-dioxane & 90 & $78: 22$ & 42 \\
\hline 10 & $4 c$ & $\mathrm{CH}_{3} \mathrm{CN}$ & 86 & $78: 22$ & 6 \\
\hline 11 & $4 c$ & EtOAc & 93 & $80: 20$ & 40 \\
\hline 12 & $4 c$ & acetone & 86 & $76: 24$ & 22 \\
\hline
\end{tabular}

${ }^{a}$ Unless otherwise indicated, the reaction was carried out at a $0.05 \mathrm{mmol}$ scale in a solvent $(0.5 \mathrm{~mL})$ at $25^{\circ} \mathrm{C}$ for $12 \mathrm{~h}$ using a 1a:2a molar ratio of 1:1.2. ${ }^{b}$ Isolated total yield of the diastereomeric mixtures. ${ }^{c}$ The diastereomeric ratio (dr) was determined by ${ }^{1} \mathrm{H}$ NMR and HPLC. ${ }^{d}$ The ee value refers to that of the major diastereomer and was determined by HPLC. The asterisk * indicates chiral center.

Table 2. Further optimization of reaction conditions ${ }^{a}$.

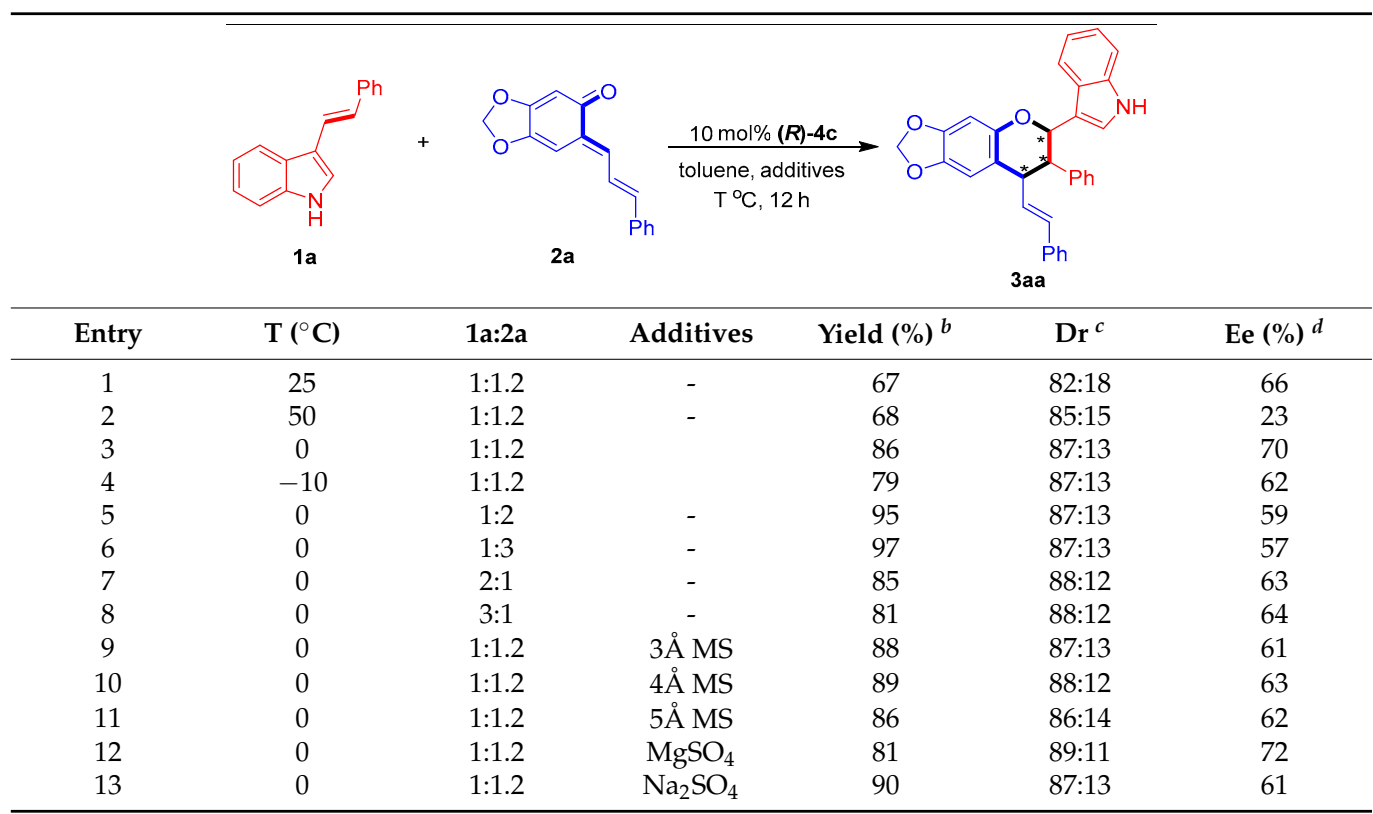

${ }^{a}$ Unless otherwise indicated, the reaction was carried out at a $0.05 \mathrm{mmol}$ scale in toluene $(0.5 \mathrm{~mL})$ with additives $(25 \mathrm{mg})$ for $12 \mathrm{~h} .{ }^{b}$ Isolated total yield of the diastereomeric mixtures. ${ }^{c}$ The diastereomeric ratio (dr) was determined by ${ }^{1} \mathrm{H}$ NMR and HPLC. ${ }^{d}$ The ee value refers to that of the major diastereomer and was determined by HPLC. The asterisk * indicates chiral center. 


\subsubsection{Substrate Scope}

After establishing the optimal reaction conditions, we investigated the substrate scope of the 3-vinylindoles 1 for catalytic asymmetric $[2+4]$ cycloadditions with sesamol-derived $o$-QM 2a. As shown in Table 3, a variety of 3-vinylindoles 1 bearing different $R / \mathrm{R}^{1}$ groups underwent $[2+4]$ cycloadditions to generate chiral indole-containing chroman derivatives 3 in overall good yields (54-98\%) and moderate to excellent stereoselectivities (78:22 dr to 93:7 dr, 55-97\% ee). In detail, C5-, C6- and C7-substituted 3-vinylindoles 1b-1f participated in the $[2+4]$ cycloaddition with high yields and moderate enantioselectivities (entries 2-6). In addition, a series of ortho-, meta- and para-substituted phenyl groups were utilized as $\mathrm{R}^{1}$ groups for the 3-vinylindoles $\mathbf{1}$, and the corresponding substrates participated in [2+4] cycloaddition with moderate to good results (entries 7-13). Among these 3-vinylindoles, 11-1m bearing para-substituted phenyl groups $\left(\mathrm{R}^{1}\right)$ delivered the corresponding products 3la-3ma with the best enantioselectivities (85\% ee and 97\% ee, entries $12-13$ ). Notably, these para-substituted substrates 11-1m displayed a much higher capability in controlling the enantioselectivity than their ortho- and meta-substituted counterparts (entries 12-13 vs. entries 7-8 and 10-11), which might be ascribed to the steric effect of the para-substituents.

Table 3. Substrate scope of 3-vinylindoles $\mathbf{1}^{a}$.

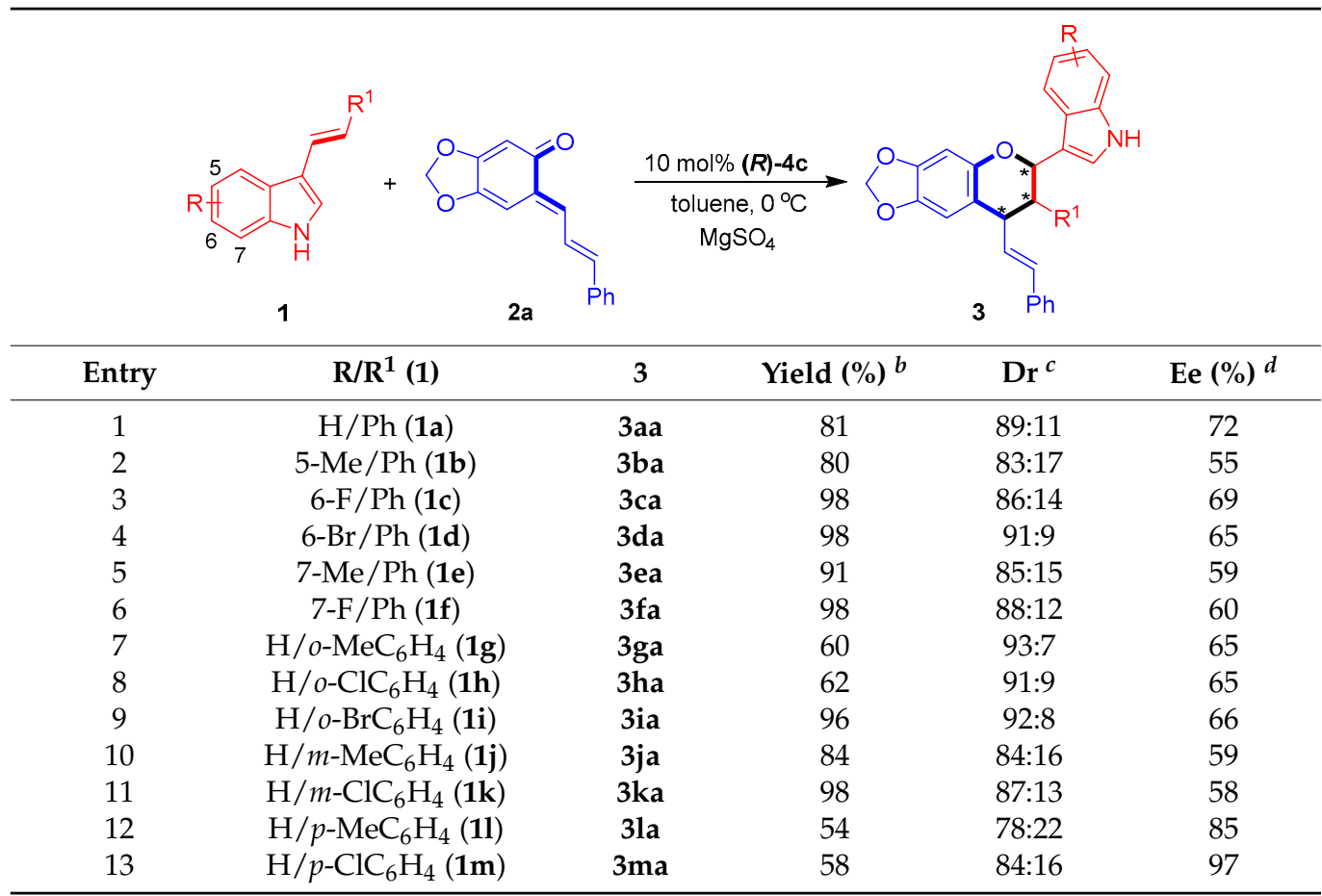

${ }^{a}$ Unless otherwise indicated, the reaction was carried out at a $0.1 \mathrm{mmol}$ scale in toluene $(1.0 \mathrm{~mL})$ with $\mathrm{MgSO}_{4}$ $\left(50 \mathrm{mg}\right.$ ) for $12 \mathrm{~h}$ using a 1:2a molar ratio of 1:1.2. ${ }^{b}$ Isolated total yield of the diastereomeric mixtures. ${ }^{c}$ The diastereomeric ratio (dr) was determined by ${ }^{1} \mathrm{H}$ NMR. ${ }^{d}$ The ee value refers to that of the major diastereomer and was determined by HPLC. The asterisk * indicates chiral center.

Next, the substrate scope of sesamol-derived $o$-QMs 2 was explored by catalytic asymmetric $[2+4]$ cycloaddition with 3-vinylindole $1 \mathbf{a}$ (Table 4$)$. This reaction was clearly amenable to participation by a series of sesamol-derived $o$-QMs $\mathbf{2 a}-\mathbf{2 g}$ bearing either electron-donating or electron-withdrawing groups at different positions of the phenyl ring (entries 1-7), producing chiral indole-containing chroman derivatives 3 in generally high yields (53-98\%) and moderate to excellent diastereo- and enantioselectivities (75:25 dr to 89:11 dr, 60-98\% ee). Among these 0 -QMs, $2 \mathbf{f}-\mathbf{2} \mathbf{g}$ bearing para-halogen-substituted phenyl groups delivered products 3af-3ag in the highest enantioselectivities of $97-98 \%$ ee (entries $4-7)$. Notably, $o$-QM $2 \mathrm{~h}$ bearing a heteroaromatic 2-thiophenyl group could also be utilized as a reaction partner to yield the product $3 \mathrm{ah}$ with a high enantioselectivity of $87 \%$ ee (entry 8). 
Table 4. Substrate scope of $o$-QMs $2^{a}$.

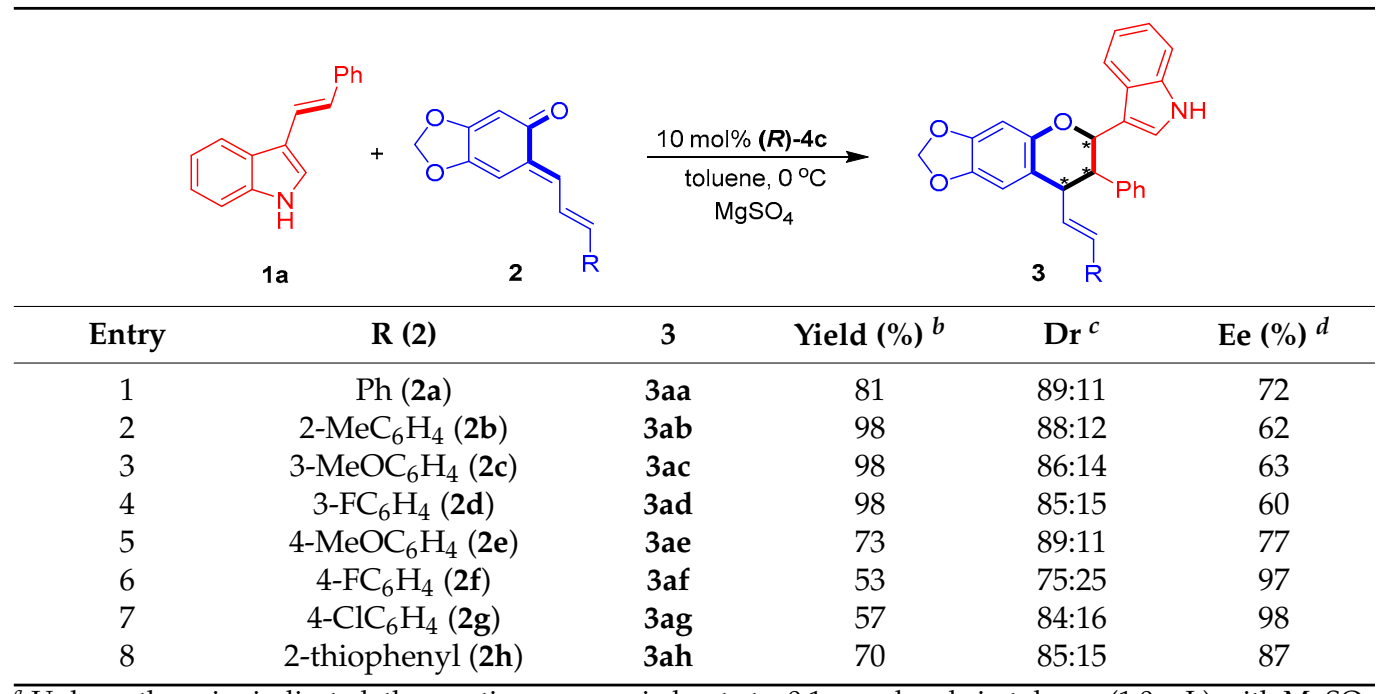

$\overline{{ }^{a}}$ Unless otherwise indicated, the reaction was carried out at a $0.1 \mathrm{mmol}$ scale in toluene $(1.0 \mathrm{~mL})$ with $\mathrm{MgSO}_{4}$ $(50 \mathrm{mg})$ for $12 \mathrm{~h}$ using a 1a:2 molar ratio of 1:1.2. ${ }^{b}$ Isolated total yield of the diastereomeric mixtures. ${ }^{c}$ The diastereomeric ratio (dr) was determined by ${ }^{1} \mathrm{H}$ NMR. ${ }^{d}$ The ee value refers to that of the major diastereomer and was determined by HPLC. The asterisk * indicates chiral center.

The structures of all products 3 were identified by their NMR, IR and HR MS data, and the ee value of all products 3 were calculated by their HPLC traces (see the Supplementary Materials). Although we tried to cultivate the single crystal from enantioenriched products 3 , we failed to achieve this goal. So, the absolute configurations of chiral products 3 could not be determined. Nevertheless, when $N$-methyl-protected 3-vinylindole 1 n was employed as a substrate in the reaction with sesamol-derived $o$-QM 2a under standard conditions (Figure 5a), the [2+4] cycloaddition occurred to generated product 3na in a moderate yield and diastereoselectivity ( $47 \%$ yield, $86: 14 \mathrm{dr}$ ) albeit with an extremely low enantioselectivity $(14 \%$ ee). Fortunately, we cultivated the single crystal of product 3 na, whose relative configuration was determined to be (trans, trans) by X-ray diffraction analysis of the single crystal (CCDC 2100427, see the Supplementary Materials) (Figure 5b).

(a) Using $\mathrm{N}$-methyl 3-vinylindole $1 \mathrm{n}$ as a substrate

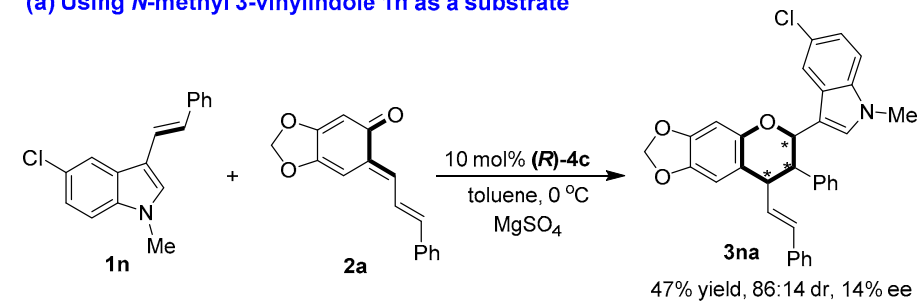

(b) Relative configuration of 3 na
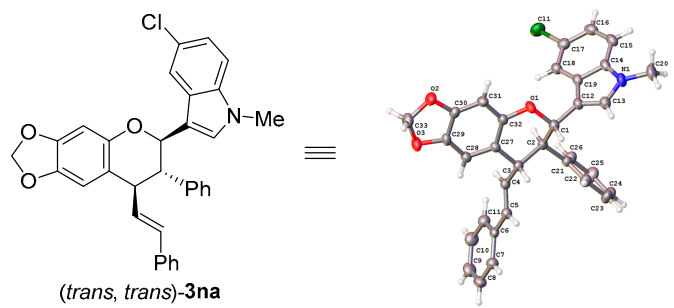

Figure 5. Determination of the relative configuration of 3na. The asterisk * indicates chiral center.

2.1.3. Theoretical Calculations of the Reaction Pathway and Key Transition States

To elucidate the reaction pathway and the interaction of CPA with the substrates, we carried out theoretical calculations on the reaction pathway of catalytic asymmetric 
$[2+4]$ cycloaddition (see the Supplementary Materials) based on previous mechanistic studies [66,67]. As exemplified by the formation of product 3ma (Figure 6a), the key transition states (TSs) and the Gibbs free energy leading to the enantiomers of 3 ma were determined, wherein TS-1 led to the major enantiomer $(R, S, R)-3$ ma and $\mathbf{T S}-\mathbf{1}^{\prime}$ led to the minor enantiomer $(S, R, S)$-3ma. DFT calculations revealed that the two bulky $3,3^{\prime}-$ (1-naphthyl) groups and the BINOL scaffold of CPA $(R)-4 \mathrm{c}$ formed a pocket-like chiral environment to hold the two substrates of $\mathbf{1 m}$ and $\mathbf{2 a}$ in a confined orientation. Specifically, in TS-1, 3-vinylindole $1 \mathrm{~m}$ was located above $o$-QM 2a in the chiral pocket of CPA $(R)-4 c$, wherein the space above $2 \mathrm{a}$ was enough to make $1 \mathrm{~m}$ have little steric effect on other groups. While in TS-1', $\mathbf{1 m}$ was located below o-QM 2a, wherein the space below 2a was limited, thus making the phenyl group of $1 \mathrm{~m}$ have some steric effect on the 1-naphthyl group of (R)-4c. This steric repulsion made TS-1' inferior to TS-1, which led to the formation of the major enantiomer $(R, S, R)$-3ma.

(a) Calculated transition states leading to the enantiomers of $3 \mathrm{ma}$

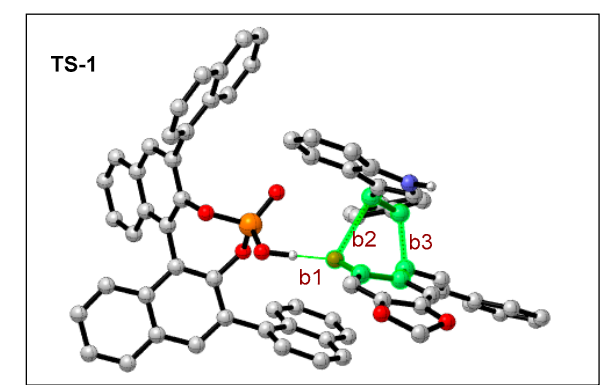

bond length: $b 1=1.461 \AA ; b 2=2.526 \AA ; b 3=1.970 \AA$

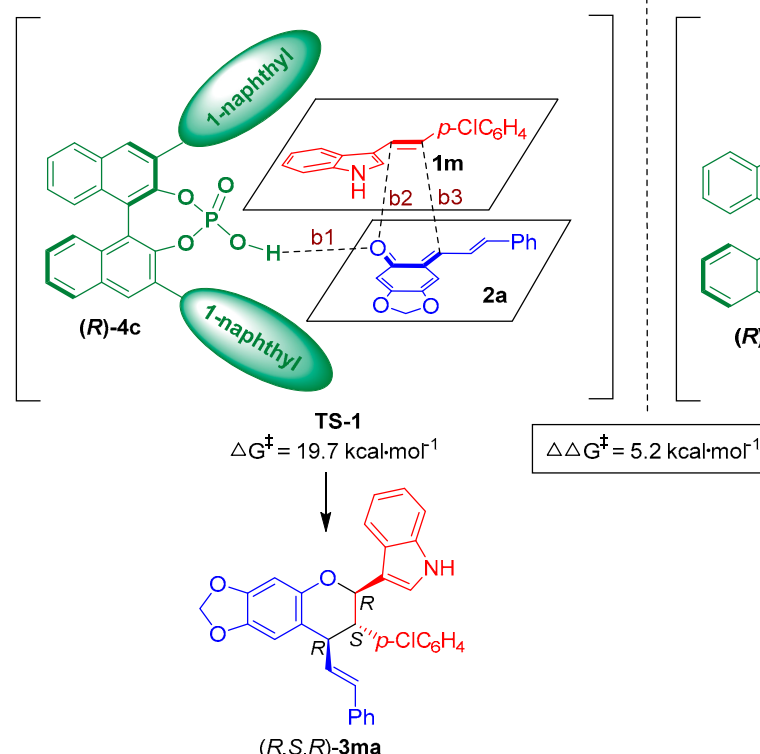

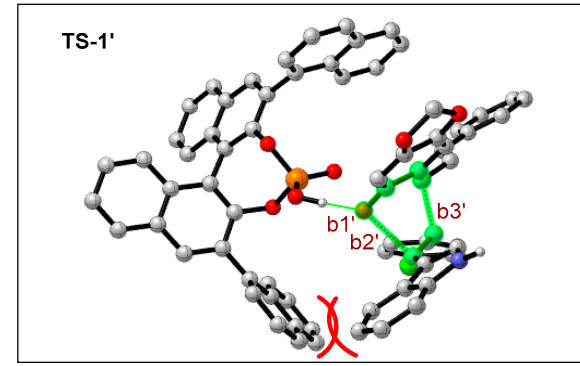

bond length: $b 1^{\prime}=1.520 \AA ; b 22^{\prime}=2.442 \AA ; b 3^{\prime}=1.977 \AA$
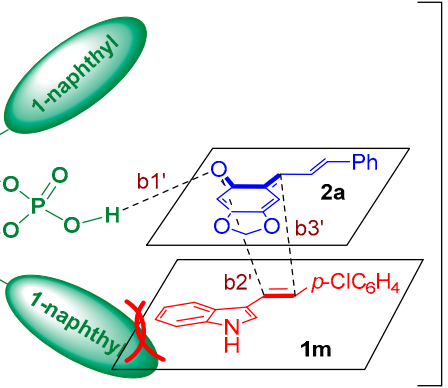

TS-1'

$\Delta \mathrm{G}^{\ddagger}=24.9 \mathrm{kcal} \cdot \mathrm{mor}^{-1}$

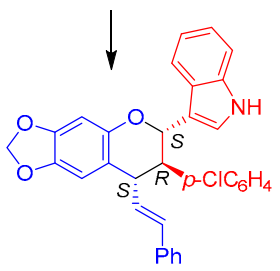

$(S, R, S)-3 m a$

(b) Control experiment to investigate the role of the $\mathrm{NH}$ group in substrate $1 \mathrm{~m}$

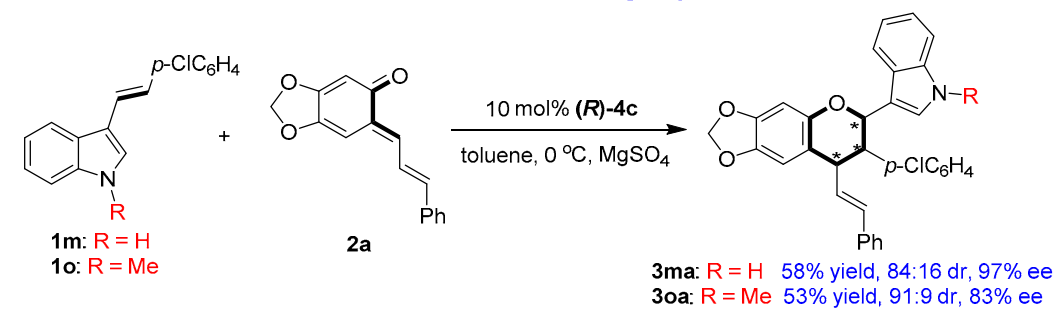

Figure 6. Calculated transition states leading to the enantiomers of $3 \mathrm{ma}$ and control experiment. The asterisk * indicates chiral center. 
In TS-1, CPA (R)-4c utilized its O-H group to form a strong hydrogen bond (b1 = $1.461 \AA$ ) with the $\mathrm{C}=\mathrm{O}$ group of $o-\mathrm{QM} 2 \mathrm{a}$, but there was no discernible hydrogen-bonding interaction between CPA $(R)-4 c$ and 3-vinylindole $1 \mathrm{~m}$. In addition, the calculations suggested that the $[2+4]$ cycloaddition largely occurred via a concerted reaction pathway involving the formation of two new bonds ( $\mathrm{b} 2=2.526 \AA$, b3 = $1.970 \AA$ ). However, the longer bond length of $\mathrm{b} 2$ than $\mathrm{b} 3$ indicated that b3 (a C-C bond) formed slightly earlier than b2 (a C-O bond), which is in accordance with the reactivity of 3-vinylindole (based on the nucleophilicity of the vinyl group). In TS-1', there were similar interactions between CPA $(R)-4 c$ and the substrates. However, the hydrogen bond $\left(\mathrm{b} 1^{\prime}=1.520 \AA\right)$ between $(R)-4 c$ and $2 \mathrm{a}$ in TS-1 was weaker than that in TS-1 $(\mathrm{b} 1=1.461 \AA)$, which resulted in a significantly higher Gibbs free energy barrier for the generation of TS-1' $(24.9 \mathrm{kcal} / \mathrm{mol})$ compared to that for TS-1 $(19.7 \mathrm{kcal} / \mathrm{mol})$. The calculated difference in the energy barriers for the two transition states of TS-1' and TS-1 of $5.2 \mathrm{kcal} / \mathrm{mol}$ explained the excellent experimentally obtained enantioselectivity of $3 \mathrm{ma}(97 \%$ ee).

Very interestingly, in the calculated transition states, there was no discernible hydrogenbonding interaction between CPA $(R)-4 c$ and 3-vinylindole $1 \mathrm{~m}$, which was seldom reported in CPA-catalyzed reactions involving 3-vinylindoles. To verify this issue, we performed a control experiment to investigate the role of the NH group in substrate 1m (Figure 6b). Namely, 3-vinylindole 1o, as $N$-methyl protected counterpart of $\mathbf{1 m}$, was employed as a substrate in the $[2+4]$ cycloaddition with $o$-QM 2a under standard conditions, which smoothly generated product $3 \mathbf{o a}$ in a moderate yield of $53 \%$ with a good diastereo- and enantioselectivity (91:9 dr, 83\% ee). Compared to the results of product $3 \mathrm{ma}$ which was generated from $N$-unprotected 3-vinylindole $\mathbf{1 m}$, the yield and the stereoselectivity of product 3oa were on a similar level, thus supporting the calculated activation mode that the NH group of 3-vinylindole $\mathbf{1 m}$ had no discernible hydrogen-bonding interaction with CPA (R)-4c.

It should be noted that the E-configuration of vinylindoles $\mathbf{1}$ has been retained as trans-configuration in products 3 due to a concerted [ +4$]$ cycloaddition pathway as illustrated in TS-1. So, the diastereomeric ratio of product 3 reflects the stereoselectivity of the two adjacent chiral centers generated by the two individual substrates $\mathbf{1}$ and $\mathbf{2}$.

\subsubsection{Large-Scale Synthesis of Product 3aa}

Finally, the catalytic asymmetric [2 +4] cycloaddition of $\mathbf{1 a}$ with $\mathbf{2 a}$ was carried out on a one mmol scale (Figure 7). The yield and stereoselectivity of this one-mmol-scale reaction were at the same level as those of the small-scale reaction (Table 3, entry 1), which implied that the catalytic asymmetric [2+4] cycloaddition could be scaled up.
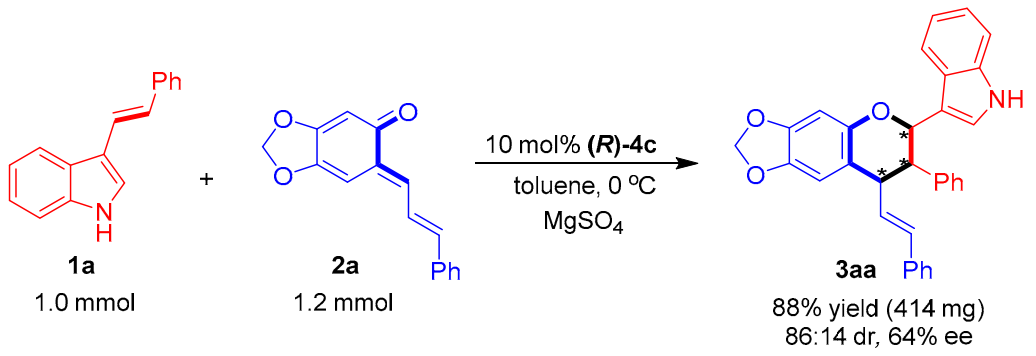

Figure 7. One-mmol-scale synthesis of product 3aa. The asterisk * indicates chiral center.

\subsection{Organocatalytic Asymmetric [2 +4] Cycloaddition of 3-Vinylindoles with} o-Hydroxybenzyl Alcohols

To expand the substrate scope of this organocatalytic asymmetric $[2+4]$ cycloaddition, we attempted to react 3-vinylindole 1a with $o$-hydroxybenzyl alcohol 5a as a precursor of $o$-QM (Table 5). In the presence of CPA (R)-4a (entry 1), the desired product 6aa was afforded in a moderate yield, albeit with a low stereoselectivity (51\% yield, 67:33 dr, 42\% ee). Then, a series of CPAs $(\boldsymbol{R})-\mathbf{4}$ were screened. Among these CPAs, $(\boldsymbol{R})-\mathbf{4} \mathbf{e}$, bearing two 
3,3'-(9-anthracenyl) groups, displayed the highest catalytic activity in delivering product 6aa with a better enantioselectivity than the other catalysts (entry 5 vs. entries 1-4 and 6-7), which could also be ascribed to the steric hindrance effect of the bulky $3,3^{\prime}$-disubstituents of CPA $(R)-4 \mathbf{e}$ in controlling the enantioselectivity. Next, different solvents were evaluated in the presence of $(\boldsymbol{R})-4 \mathbf{e}$, revealing toluene to still be the most suitable solvent (entry 5 vs. entries 8-12). Finally, the reaction temperature was modulated (entries 13-15), and the optimal reaction conditions were set as shown in entry 14 .

Table 5. Optimization of reaction conditions for [2+4] cycloaddition of $\mathbf{1 a}$ with $\mathbf{5 a}{ }^{a}$.

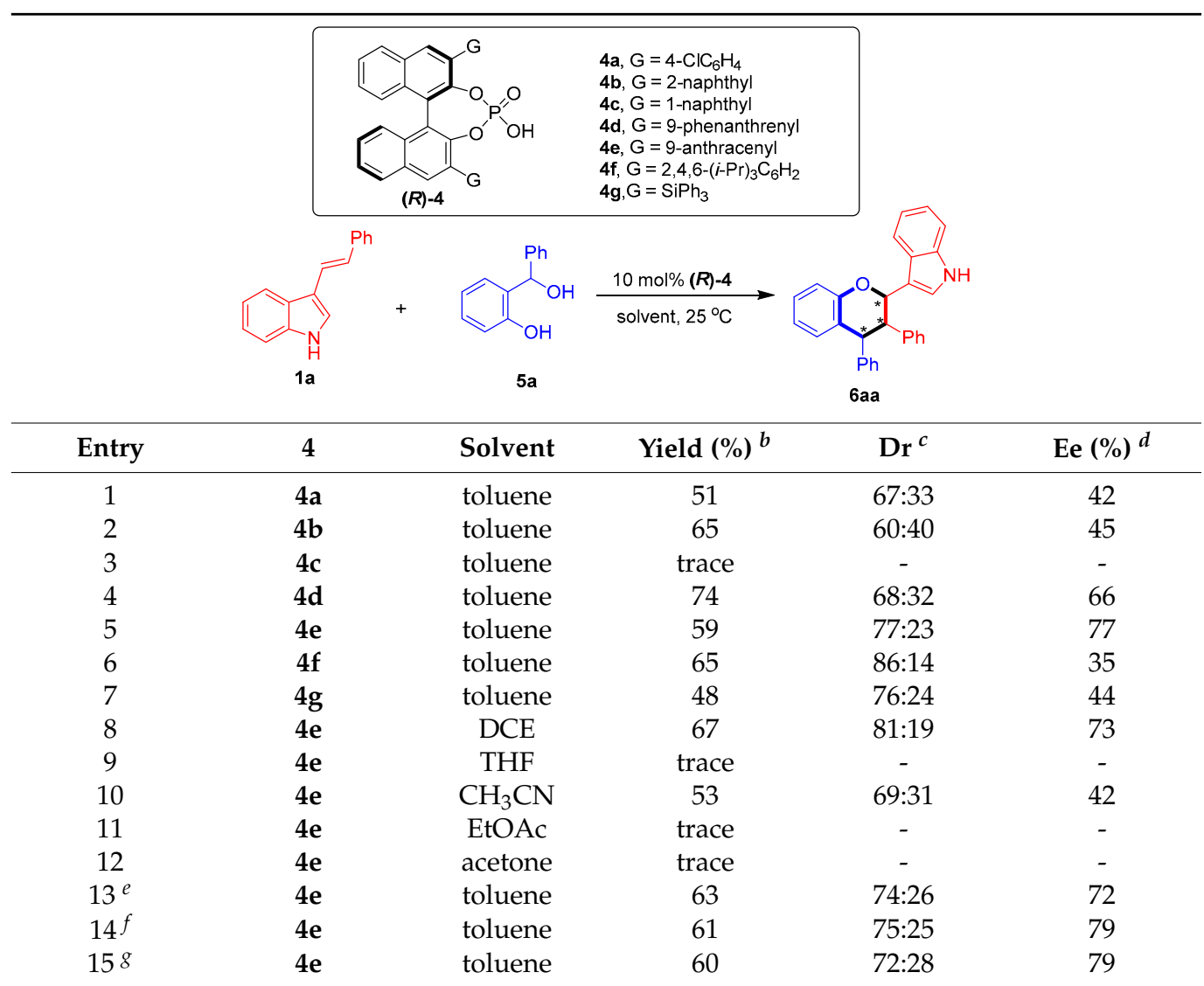

${ }^{a}$ Unless otherwise indicated, the reaction was carried out at a $0.1 \mathrm{mmol}$ scale in a solvent $(0.1 \mathrm{~mL})$ at $25^{\circ} \mathrm{C}$ for $6 \mathrm{~h}$ using a 1a:5a molar ratio of 1:1.2. ${ }^{b}$ Isolated total yield of the diastereomeric mixtures. ${ }^{c}$ The diastereomeric ratio (dr) was determined by ${ }^{1} \mathrm{H}$ NMR and HPLC. ${ }^{d}$ The ee value refers to that of the major diastereomer and was determined by HPLC. ${ }^{e}$ At $50{ }^{\circ} \mathrm{C} .{ }^{f}$ At $0{ }^{\circ} \mathrm{C} .{ }^{g}$ At $-10^{\circ} \mathrm{C}$. The asterisk ${ }^{*}$ indicates chiral center.

With the optimal conditions in hand, we investigated the substrate scope of 3-vinylindole $\mathbf{1}$ in catalytic asymmetric [2+4] cycloaddition with the $o$-hydroxybenzyl alcohol $\mathbf{5 a}$. As shown in Table 6, this [2+4] cycloaddition was amenable to participation by a wide range of 3-vinylindoles 1 bearing different $R / R^{1}$ groups. In detail, C5-, C6- and C7-substituted 3 -vinylindoles participated in the $[2+4]$ cycloaddition with the $o$-hydroxybenzyl alcohol 5 a to generate the chiral indole-containing chroman derivatives 6 in moderate to good diastereo- and enantioselectivities (75:25 dr to 83:17 dr, 74-82\% ee, entries 2-7). In addition, meta- and para-substituted phenyl groups were found to be suitable $\mathrm{R}^{1}$ groups for the 3 -vinylindoles 1 , and the corresponding substrates participated in [2+4] cycloaddition with good results (entries 8-9). 
Table 6. Substrate scope of 3-vinylindoles $\mathbf{1}$ for [2+4] cycloaddition with $o$-hydroxybenzyl alcohol $\mathbf{5 a}{ }^{a}$.

\begin{tabular}{|c|c|c|c|c|c|}
\hline & $\underbrace{R^{1}}$ & & $\frac{R)-4 \mathrm{e}}{0^{\circ} \mathrm{C}}$ & & \\
\hline Entry & $R / R^{1}(1)$ & 6 & Yield (\%) $b$ & $\mathrm{Dr}^{c}$ & $\operatorname{Ee}(\%)^{d}$ \\
\hline 1 & $\mathrm{H} / \mathrm{Ph}(\mathbf{1 a})$ & 6aa & 61 & $77: 23$ & 79 \\
\hline 2 & $5-\mathrm{Me} / \mathrm{Ph}(\mathbf{1 b})$ & $6 \mathrm{ba}$ & 50 & $75: 25$ & 76 \\
\hline 3 & 6-Cl/Ph (1p) & $6 \mathrm{pa}$ & 72 & $75: 25$ & 74 \\
\hline 4 & 6-Br/Ph (1d) & $6 \mathrm{da}$ & 77 & $78: 22$ & 74 \\
\hline 5 & 7-Me/Ph (1e) & 6ea & 53 & $75: 25$ & 74 \\
\hline 6 & 7-Cl/Ph (1q) & 6qa & 90 & $82: 18$ & 75 \\
\hline 7 & 7-Br/Ph (1r) & 6ra & 89 & $83: 17$ & 82 \\
\hline 8 & $\mathrm{H} / m-\mathrm{ClC}_{6} \mathrm{H}_{4}(\mathbf{1 k})$ & $6 \mathrm{ka}$ & 72 & $76: 24$ & 80 \\
\hline 9 & $\mathrm{H} / p-\mathrm{ClC}_{6} \mathrm{H}_{4}(\mathbf{1 m})$ & $6 \mathrm{ma}$ & 70 & $78: 22$ & 83 \\
\hline
\end{tabular}

${ }^{a}$ Unless otherwise indicated, the reaction was carried out at a $0.1 \mathrm{mmol}$ scale in toluene $(1.0 \mathrm{~mL})$ for $6 \mathrm{~h}$ using a 1:5a molar ratio of 1:1.2. ${ }^{b}$ Isolated total yield of the diastereomeric mixtures. ${ }^{c}$ The diastereomeric ratio (dr) was determined by ${ }^{1} \mathrm{H}$ NMR. ${ }^{d}$ The ee value refers to that of the major diastereomer and was determined by HPLC. The asterisk * indicates chiral center.

Then, the substrate scope of $o$-hydroxybenzyl alcohols 5 was investigated for [2+4] cycloaddition with 3-vinylindole 1a under standard reaction conditions. As shown in Table 7 , the $o$-hydroxybenzyl alcohols $\mathbf{5 b} \mathbf{b} \mathbf{- 5}$ bearing a methyl group or a halogen group at the $\mathrm{C} 5$ position successfully participated in [2+4] cycloaddition with 3-vinylindole $\mathbf{1 a}$, providing products $\mathbf{6 a b}-\mathbf{6 a c}$ in moderate to good diastereo- and enantioselectivities (68:32 dr to $81: 19 \mathrm{dr}, 73-76 \%$ ee, entries $2-3$ ). In addition, aromatic $\mathrm{R}^{1}$ groups with ortho, meta and para-substituents were successfully employed in the reaction, affording products 6ad-6af in overall good enantioselectivities (76-81\% ee, entries $4-6)$.

Table 7. Substrate scope of $o$-hydroxybenzyl alcohols 5 for $[2+4]$ cycloaddition ${ }^{a}$.

\begin{tabular}{|c|c|c|c|c|c|}
\hline & 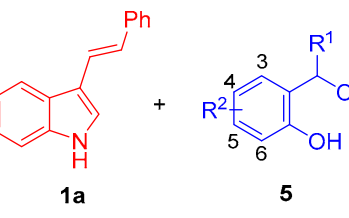 & $\frac{10 \mathrm{mc}}{\text { tolue }}$ & & & \\
\hline Entry & $\mathbf{R}^{1 / \mathbf{R}^{2}}(5)$ & 6 & Yield (\%) $b$ & $\mathrm{Dr}^{c}$ & $\operatorname{Ee}(\%)^{d}$ \\
\hline 1 & $\mathrm{Ph} / \mathrm{H}(\mathbf{5 a})$ & 6aa & 61 & $77: 23$ & 79 \\
\hline 2 & $\mathrm{Ph} / 5-\mathrm{Me}(5 \mathbf{b})$ & $6 a b$ & 73 & $81: 19$ & 76 \\
\hline $3^{e}$ & $\mathrm{Ph} / 5-\mathrm{Br}(5 \mathrm{c})$ & 6ac & 57 & $68: 32$ & $73(60)^{f}$ \\
\hline 4 & $p-\mathrm{FC}_{6} \mathrm{H}_{4} / \mathrm{H}(\mathbf{5 d})$ & 6ad & 59 & $75: 25$ & 76 \\
\hline 5 & $m-\mathrm{MeOC}_{6} \mathrm{H}_{4} / \mathrm{H}(\mathbf{5 e})$ & 6ae & 80 & $75: 25$ & 79 \\
\hline $6^{g}$ & $o-\mathrm{MeOC}_{6} \mathrm{H}_{4} / 4-\mathrm{OMe}(\mathbf{5 f})$ & 6af & 71 & $90: 10$ & 81 \\
\hline
\end{tabular}

${ }^{a}$ Unless otherwise indicated, the reaction was carried out at a $0.1 \mathrm{mmol}$ scale in toluene (1.0 $\left.\mathrm{mL}\right)$ for $6 \mathrm{~h}$ using a 1a:5 molar ratio of $1: 1.2 .^{b}$ Isolated total yield of the diastereomeric mixtures. ${ }^{c}$ The diastereomeric ratio (dr) was determined by ${ }^{1} \mathrm{H}$ NMR. ${ }^{d}$ The ee value refers to that of the major diastereomer and was determined by HPLC. ${ }^{e}$ At $0{ }^{\circ} \mathrm{C}$ for $6 \mathrm{~h}$ and then $25^{\circ} \mathrm{C}$ for $2 \mathrm{~h}$. ${ }^{f}$ The ee value of the minor diastereoisomer. ${ }^{g}$ At $25^{\circ} \mathrm{C}$ for $2 \mathrm{~h}$. The asterisk * indicates chiral center.

The structures of all products 6 were identified by their NMR, IR and HR MS data, and the ee value of all products 6 were calculated by their HPLC traces (see the Supplementary Materials). The relative configuration of product $\mathbf{6 m a}$ was determined to be (trans, cis) by 
a NOE experiment (see the Supplementary Materials) (Figure 8) and comparing the ${ }^{1} \mathrm{H}$ NMR spectra with that of a similar compound [18].

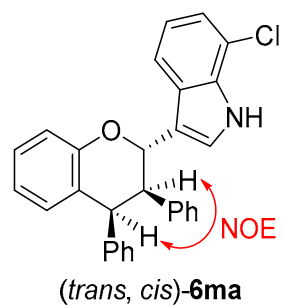

Figure 8. Relative configuration of $6 \mathrm{ma}$.

\section{Materials and Methods}

The detailed procedures for the synthesis and characterization of the products are given in Appendix A section.

\section{Conclusions}

In summary, we performed catalytic asymmetric $[2+4]$ cycloaddition of 3-vinylindoles with ortho-quinone methides and their precursors in the presence of chiral phosphoric acid. This approach was used to synthesize a series of indole-containing chroman derivatives with structural diversity in overall high yields (up to $98 \%$ ), good diastereoselectivities (up to $93: 7 \mathrm{dr}$ ) and moderate to excellent enantioselectivities (up to $98 \%$ ee). This approach not only enriches the chemistry of 3-vinylindole-inolved catalytic asymmetric cycloadditions but is also useful for the enantioselective synthesis of chiral chroman derivatives.

Supplementary Materials: The following are available online. NMR and HPLC spectra of products 3 and 6 , NOE spectrum of product $6 \mathrm{ma}$, X-ray single-crystal data for product 3na, and theoretical calculations of the reaction pathway.

Author Contributions: Conceptualization, F.S.; Data curation, Y.-C.Z.; Investigation, S.-J.L., M.-S.T., K.-Y.L. and J.-Y.C.; Supervision, Y.-C.Z. and F.S.; Writing—original draft, Y.-C.Z.; Writing-review \& editing, S.-F.N. and F.S. All authors have read and agreed to the published version of the manuscript.

Funding: This work was supported by National Natural Science Foundation of China (22125104 and 21831007), Natural Science Foundation of Jiangsu Province (BK20201018), Natural Science Foundation of Xuzhou City (KC21021), High-level Innovative and Entrepreneurial Talents Introduction Plan of Jiangsu Province and Natural Science Foundation of JSNU (19XSRX010).

Institutional Review Board Statement: Not applicable.

Informed Consent Statement: Not applicable.

Data Availability Statement: The data presented in this study are available in this article.

Conflicts of Interest: The authors declare no conflict of interest.

Sample Availability: Samples of the compounds are not available from the authors.

\section{Appendix A Experimental Section}

${ }^{1} \mathrm{H}$ and ${ }^{13} \mathrm{C}$ NMR spectra were measured at 400 and $100 \mathrm{MHz}$, respectively. The solvents used for NMR spectroscopy were acetone- $d_{6}$ and $\mathrm{CDCl}_{3}$, using tetramethylsilane as the internal reference. HR MS (ESI) was determined by an HR MS/MS instrument. The X-ray source used for the single crystal X-ray diffraction analysis of compound 3na was $\operatorname{MoK} \alpha(\lambda=0.71073)$, and the thermal ellipsoid was drawn at the $30 \%$ probability level. Analytical grade solvents for the column chromatography were used after distillation, and commercially available reagents were used as received. Substrates 1 were synthesized according to the literature method [17]. Substrates $\mathbf{2}$ and $\mathbf{5}$ were synthesized according to the literature method $[52,68]$. 


\section{General procedure for the synthesis of products 3}

To the mixture of 3-vinylindoles $1(0.1 \mathrm{mmol})$, ortho-quinone methides $2(0.12 \mathrm{mmol})$, catalyst $(\boldsymbol{R})-4 \mathrm{c}(0.01 \mathrm{mmol})$, and $\mathrm{MgSO}_{4}(50 \mathrm{mg})$ was added toluene $(1 \mathrm{~mL})$. Then, the reaction mixture was stirred at $0{ }^{\circ} \mathrm{C}$ for $12 \mathrm{~h}$. After completion of the reaction, which was indicated by TLC, the reaction mixture was directly purified through flash column chromatography to afford products 3 .

(E)-2-(7-phenyl-8-styryl-7,8-dihydro-6H-[1,3]dioxolo[4,5-g]chromen-6-yl)-1H-indole (3aa): Yield: $81 \%$ (38.0 mg); 89:11 dr; white solid; m.p. $97.6-99.0{ }^{\circ} \mathrm{C} ;[\alpha]_{\mathrm{D}}{ }^{20}=-19.0$ (c 0.76 , acetone); ${ }^{1} \mathrm{H}$ NMR $\left(400 \mathrm{MHz}, \mathrm{CDCl}_{3}\right) \delta 7.88-7.80(\mathrm{~m}, 2 \mathrm{H}), 7.26-7.22(\mathrm{~m}, 5 \mathrm{H}), 7.20-7.18(\mathrm{~m}$, $1 \mathrm{H}), 7.17-7.12(\mathrm{~m}, 2 \mathrm{H}), 7.07(\mathrm{~m}, 2 \mathrm{H}), 7.03-7.00(\mathrm{~m}, 1 \mathrm{H}), 7.00-6.96(\mathrm{~m}, 2 \mathrm{H}), 6.79(\mathrm{~d}, J=2.4 \mathrm{~Hz}$, $1 \mathrm{H}), 6.77(\mathrm{~s}, 1 \mathrm{H}), 6.56(\mathrm{~s}, 1 \mathrm{H}), 6.16(\mathrm{~d}, J=15.7 \mathrm{~Hz}, 1 \mathrm{H}), 6.06-5.96(\mathrm{~m}, 1 \mathrm{H}), 5.92-5.89(\mathrm{~m}$, $2 \mathrm{H}), 5.48(\mathrm{~d}, J=10.5 \mathrm{~Hz}, 1 \mathrm{H}), 4.01-3.95(\mathrm{~m}, 1 \mathrm{H}), 3.57-3.50(\mathrm{~m}, 1 \mathrm{H}) .{ }^{13} \mathrm{C}$ NMR $(100 \mathrm{MHz}$, $\left.\mathrm{CDCl}_{3}\right) \delta 149.8,147.0,141.7,140.9,137.1,136.2,132.8,131.3,128.4,128.3,128.1,127.2,126.4$, $126.2,125.9,123.3,122.2,120.1,119.8,116.4,114.4,111.2,108.2,101.0,98.8,76.9,50.5,48.2$; IR (KBr): 3419, 3057, 3026, 2894, 1499, 1477, 1264, 1153, 1072, $864 \mathrm{~cm}^{-1} ;\left(\mathrm{C}_{32} \mathrm{H}_{25} \mathrm{NO}_{3}-\mathrm{H}\right)^{-}$ requires $m / z 470.1761$, found $m / z$ 470.1749; ee: $72 \%, \mathrm{AD}-\mathrm{H}$, hexane/isopropanol $=70 / 30$, $\mathrm{t}_{\mathrm{R}}=11.147$ (minor), $\mathrm{t}_{\mathrm{R}}=30.360$ (major).

(E)-5-methyl-2-(7-phenyl-8-styryl-7,8-dihydro-6H-[1,3]dioxolo[4,5-g]chromen-6-yl)-1Hindole (3ba):

Yield: $80 \%$ (38.9 mg); 83:17 dr; white solid; m.p. $123.2-125.3^{\circ} \mathrm{C} ;[\alpha]_{\mathrm{D}}{ }^{20}=-26.1$ (c 0.78 , acetone); ${ }^{1} \mathrm{H} \mathrm{NMR}\left(400 \mathrm{MHz}, \mathrm{CDCl}_{3}\right) \delta 7.68(\mathrm{~s}, 1 \mathrm{H}), 7.61(\mathrm{~s}, 1 \mathrm{H}), 7.30-7.27(\mathrm{~m}, 1 \mathrm{H}), 7.26-7.17$ $(\mathrm{m}, 4 \mathrm{H}), 7.13-7.06(\mathrm{~m}, 3 \mathrm{H}), 7.05-6.98(\mathrm{~m}, 4 \mathrm{H}), 6.80(\mathrm{~s}, 1 \mathrm{H}), 6.72(\mathrm{~d}, J=2.5 \mathrm{~Hz}, 1 \mathrm{H}), 6.60(\mathrm{~s}$, $1 \mathrm{H}), 6.20-6.16(\mathrm{~m}, 1 \mathrm{H}), 6.09-6.00(\mathrm{~m}, 1 \mathrm{H}), 5.92(\mathrm{~s}, 2 \mathrm{H}), 5.45(\mathrm{~d}, J=10.5 \mathrm{~Hz}, 1 \mathrm{H}), 4.02-3.96(\mathrm{~m}$, 1H), 3.59-3.50 (m, 1H), $2.49(\mathrm{~s}, 3 \mathrm{H}) ;{ }^{13} \mathrm{C}$ NMR $\left(100 \mathrm{MHz}, \mathrm{CDCl}_{3}\right) \delta$ 149.8, 147.0, 141.7, 141.1, 137.2, 134.6, 132.8, 131.4, 129.2, 129.0, 128.5, 128.4, 128.3, 128.1, 128.0, 127.2, 126.5, 126.4, 126.3, 126.2, 123.8, 123.5, 119.6, 116.4, 113.7, 110.9, 108.2, 101.0, 98.9, 77.0, 50.3, 48.3, 21.6; IR (KBr): 3416, 3025, 2895, 1499, 1478, 1425, 1238, 1152, 1037, $748 \mathrm{~cm}^{-1} ;\left(\mathrm{C}_{33} \mathrm{H}_{27} \mathrm{NO}_{3}-\mathrm{H}\right)^{-}$ requires $m / z 484.1918$, found $m / z 484.1890$; ee: $55 \%$, IA, hexane/isopropanol $=70 / 30$, $\mathrm{t}_{\mathrm{R}}=10.293$ (minor), $\mathrm{t}_{\mathrm{R}}=30.297$ (major).

(E)-6-fluoro-2-(7-phenyl-8-styryl-7,8-dihydro-6H-[1,3]dioxolo[4,5-g]chromen-6-yl)- $1 H$ indole (3ca):

Yield: $98 \%$ (47.8 mg); 86:14 dr; brown solid; m.p. 108.7-110.0 ${ }^{\circ} \mathrm{C} ;[\alpha]_{\mathrm{D}}{ }^{20}=-27.8$ (c 0.96 , acetone); ${ }^{1} \mathrm{H}$ NMR (400 MHz, $\left.\mathrm{CDCl}_{3}\right) \delta$ 7.77-7.70 (m, 2H), 7.29-7.26 (m, 1H), 7.25-7.22 (m, $3 \mathrm{H}), 7.20-7.16(\mathrm{~m}, 1 \mathrm{H}), 7.12-7.06(\mathrm{~m}, 2 \mathrm{H}), 7.06-7.01(\mathrm{~m}, 1 \mathrm{H}), 7.00-6.95(\mathrm{~m}, 2 \mathrm{H}), 6.91-6.84$ $(\mathrm{m}, 2 \mathrm{H}), 6.78(\mathrm{~s}, 1 \mathrm{H}), 6.73(\mathrm{~d}, J=2.1 \mathrm{~Hz}, 1 \mathrm{H}), 6.57(\mathrm{~s}, 1 \mathrm{H}), 6.18(\mathrm{~d}, J=15.5 \mathrm{~Hz}, 1 \mathrm{H}), 6.05-5.97$ $(\mathrm{m}, 1 \mathrm{H}), 5.92-5.89(\mathrm{~m}, 2 \mathrm{H}), 5.43(\mathrm{~d}, J=10.5 \mathrm{~Hz}, 1 \mathrm{H}), 4.03-3.95(\mathrm{~m}, 1 \mathrm{H}), 3.53-3.43(\mathrm{~m}, 1 \mathrm{H})$; ${ }^{13} \mathrm{C}$ NMR $\left(100 \mathrm{MHz}, \mathrm{CDCl}_{3}\right) \delta 159.9(\mathrm{~d}, J=236.0 \mathrm{~Hz}), 149.6,147.0,141.8,140.8,137.1$, $136.1(J=12.0 \mathrm{~Hz}), 132.9,131.1,128.4,128.3,128.2,127.3,126.5,126.2,123.7(\mathrm{~d}, J=2.0$ $\mathrm{Hz}), 122.4,120.9$ (d, J = 10.0 Hz), 116.4, 114.5, $108.6(\mathrm{~d}, J=24.0 \mathrm{~Hz}), 108.2,101.0,98.7,97.5$ $(\mathrm{d}, J=26.0 \mathrm{~Hz}), 76.9,50.7,48.1$; IR (KBr): 3891, 3725, 3421, 1844, 1699, 1239, 1153, 862, $747 \mathrm{~cm}^{-1} ;\left(\mathrm{C}_{32} \mathrm{H}_{24} \mathrm{FNO}_{3}-\mathrm{H}\right)^{-}$requires $\mathrm{m} / \mathrm{z}$ 488.1667, found $\mathrm{m} / \mathrm{z}$ 488.1676; ee: $69 \%$, IA, hexane/isopropanol $=70 / 30, \mathrm{t}_{\mathrm{R}}=9.773$ (major), $\mathrm{t}_{\mathrm{R}}=25.597$ (minor).

(E)-6-bromo-2-(7-phenyl-8-styryl-7,8-dihydro-6H-[1,3]dioxolo[4,5-g]chromen-6-yl)-1Hindole (3da):

Yield: $98 \%$ (53.8 mg); $91: 9 \mathrm{dr}$; brown solid; m.p. $104.5-105.1{ }^{\circ} \mathrm{C}$; $[\alpha]_{\mathrm{D}}{ }^{20}=-18.2$ (c 1.08, acetone); ${ }^{1} \mathrm{H}$ NMR $\left(400 \mathrm{MHz}, \mathrm{CDCl}_{3}\right) \delta 7.73(\mathrm{~s}, 1 \mathrm{H}), 7.67(\mathrm{~d}, J=8.5 \mathrm{~Hz}, 1 \mathrm{H}), 7.32-7.26(\mathrm{~m}$, 2H), 7.25-7.23 (m, 3H), 7.22-7.21 (m, 1H), 7.21-7.16 (m, 1H), 7.12-7.06 (m, 2H), 7.05-7.00 $(\mathrm{m}, 1 \mathrm{H}), 6.99-6.93(\mathrm{~m}, 2 \mathrm{H}), 6.78(\mathrm{~s}, 1 \mathrm{H}), 6.71-6.67(\mathrm{~m}, 1 \mathrm{H}), 6.55(\mathrm{~s}, 1 \mathrm{H}), 6.18(\mathrm{~d}, J=15.7$ $\mathrm{Hz}, 1 \mathrm{H}), 6.05-5.96(\mathrm{~m}, 1 \mathrm{H}), 5.92-5.88(\mathrm{~m}, 2 \mathrm{H}), 5.42(\mathrm{~d}, J=10.5 \mathrm{~Hz}, 1 \mathrm{H}), 4.01-3.94(\mathrm{~m}, 1 \mathrm{H})$, 3.50-3.41 (m, 1H); ${ }^{13} \mathrm{C}$ NMR (100 MHz, $\left.\mathrm{CDCl}_{3}\right) \delta 149.5,147.0,141.9,140.7,137.0,136.9$, 133.0, 131.0, 128.4, 128.3, 127.3, 126.6, 126.2, 124.7, 123.9, 123.1, 121.3, 116.4, 115.8, 114.6, 114.2, 108.2, 101.1, 98.7, 76.8, 50.7, 48.1; IR (KBr): 3801, 3735, 3670, 3421, 1844, 1576, 1670, 
964, 861, $747 \mathrm{~cm}^{-1} ;\left(\mathrm{C}_{32} \mathrm{H}_{24} \mathrm{BrNO}_{3}-\mathrm{H}\right)^{-}$requires $\mathrm{m} / z$ 548.0867, found $\mathrm{m} / \mathrm{z}$ 548.0844; ee: $65 \%$, IA, hexane/isopropanol $=70 / 30, \mathrm{t}_{\mathrm{R}}=13.480$ (minor), $\mathrm{t}_{\mathrm{R}}=28.633$ (major).

(E)-7-methyl-2-(7-phenyl-8-styryl-7,8-dihydro-6H-[1,3]dioxolo[4,5-g]chromen-6-yl)-1Hindole (3ea):

Yield: 91\% (44.1 mg); 85:15 dr; brown solid; m.p. $106.3-107.5{ }^{\circ} \mathrm{C} ;[\alpha]_{\mathrm{D}}{ }^{20}=-20.0$ (c 0.88 , acetone); ${ }^{1} \mathrm{H}$ NMR (400 MHz, $\left.\mathrm{CDCl}_{3}\right) \delta 7.71-7.62(\mathrm{~m}, 2 \mathrm{H}), 7.25-7.16(\mathrm{~m}, 5 \mathrm{H}), 7.13-7.04$ $(\mathrm{m}, 3 \mathrm{H}), 7.04-7.00(\mathrm{~m}, 3 \mathrm{H}), 6.96(\mathrm{~d}, J=7.0 \mathrm{~Hz}, 1 \mathrm{H}), 6.82-6.75(\mathrm{~m}, 2 \mathrm{H}), 6.57-6.54(\mathrm{~m}, 1 \mathrm{H})$, $6.16(\mathrm{~d}, J=16.6 \mathrm{~Hz}, 1 \mathrm{H}), 6.06-5.98(\mathrm{~m}, 1 \mathrm{H}), 5.91(\mathrm{~s}, 2 \mathrm{H}), 5.49(\mathrm{~d}, J=10.5 \mathrm{~Hz}, 1 \mathrm{H}), 4.00-3.94$ $(\mathrm{m}, 1 \mathrm{H}), 3.60-3.50(\mathrm{~m}, 1 \mathrm{H}), 2.35-2.29(\mathrm{~m}, 3 \mathrm{H}) ;{ }^{13} \mathrm{C} \mathrm{NMR}\left(100 \mathrm{MHz}, \mathrm{CDCl}_{3}\right) \delta 149.8,146.9$, $141.7,141.1,137.1,135.8,132.8,131.3,128.4,128.3,128.2,127.2,126.4,126.2,125.4,123.1$, 122.7, 120.3, 120.0, 117.8, 116.4, 114.8, 108.2, 101.0, 98.8, 76.8, 50.4, 48.4, 16.5; IR (KBr): 3853, $3751,3735,3711,1734,1684,1476,1152,1038,748 \mathrm{~cm}^{-1} ;\left(\mathrm{C}_{33} \mathrm{H}_{27} \mathrm{NO}_{3}-\mathrm{H}\right)^{-}$requires $m / z$ 484.1918, found $\mathrm{m} / \mathrm{z}$ 484.1893; ee: $59 \%$, IA, hexane $/$ isopropanol $=90 / 10, \mathrm{t}_{\mathrm{R}}=55.313$ (minor), $\mathrm{t}_{\mathrm{R}}=117.610$ (major).

(E)-7-fluoro-2-(7-phenyl-8-styryl-7,8-dihydro-6H-[1,3]dioxolo[4,5-g]chromen-6-yl)-1Hindole (3fa):

Yield: $98 \%$ (47.9 mg); 88:12 dr; brown solid; m.p. $78.2-79.0{ }^{\circ} \mathrm{C} ;[\alpha]_{\mathrm{D}}{ }^{20}=-30.3(\mathrm{c} 0.96$, acetone); ${ }^{1} \mathrm{H} \mathrm{NMR}\left(400 \mathrm{MHz}, \mathrm{CDCl}_{3}\right) \delta 8.03(\mathrm{~s}, 1 \mathrm{H}), 7.58(\mathrm{~d}, J=8.0 \mathrm{~Hz}, 1 \mathrm{H}), 7.25-7.14(\mathrm{~m}$, $5 \mathrm{H}), 7.12-7.07(\mathrm{~m}, 2 \mathrm{H}), 7.05-7.00(\mathrm{~m}, 2 \mathrm{H}), 7.00-6.96(\mathrm{~m}, 2 \mathrm{H}), 6.91-6.86(\mathrm{~m}, 1 \mathrm{H}), 6.82(\mathrm{~d}$, $J=2.4 \mathrm{~Hz}, 1 \mathrm{H}), 6.78(\mathrm{~s}, 1 \mathrm{H}), 6.57(\mathrm{~s}, 1 \mathrm{H}), 6.20-6.16(\mathrm{~m}, 1 \mathrm{H}), 6.06-5.97(\mathrm{~m}, 1 \mathrm{H}), 5.92-5.89(\mathrm{~m}$, $2 \mathrm{H}), 5.45(\mathrm{~d}, J=10.5 \mathrm{~Hz}, 1 \mathrm{H}), 4.03-3.96(\mathrm{~m}, 1 \mathrm{H}), 3.55-3.45(\mathrm{~m}, 1 \mathrm{H}) ;{ }^{13} \mathrm{C} \mathrm{NMR}(100 \mathrm{MHz}$, $\left.\mathrm{CDCl}_{3}\right) \delta 148.4(\mathrm{~d}, J=261.0 \mathrm{~Hz}), 141.9,140.7,137.1,133.0,131.1,128.4(\mathrm{~d}, J=18.0 \mathrm{~Hz}), 128.3$, $127.3,126.6,126.2,124.0,120.2(\mathrm{~d}, J=6.0 \mathrm{~Hz}), 116.4,116.0(\mathrm{~d}, J=3.0 \mathrm{~Hz}), 115.4,108.3,107.1$ $(\mathrm{d}, J=16.0 \mathrm{~Hz}), 101.1,98.8,76.8,50.7,48.2$; IR (KBr): 3779, 3702, 3689, 3675, 3567, 1869, 1700, $1559,1039,748 \mathrm{~cm}^{-1} ;\left(\mathrm{C}_{32} \mathrm{H}_{24} \mathrm{FNO}_{3}-\mathrm{H}\right)^{-}$requires $\mathrm{m} / \mathrm{z} 488.1667$, found $\mathrm{m} / \mathrm{z} 488.1672$; ee: $60 \%$, IA, hexane/isopropanol $=70 / 30, \mathrm{t}_{\mathrm{R}}=11.473$ (major), $\mathrm{t}_{\mathrm{R}}=14.520$ (minor).

(E)-2-(8-styryl-7-(o-tolyl)-7,8-dihydro-6H-[1,3]dioxolo[4,5-g]chromen-6-yl)-1H-indole (3ga): Yield: 60\% (29.2 mg); 93:7 dr; brown solid; m.p. $181.2-182.9{ }^{\circ} \mathrm{C} ;[\alpha]_{\mathrm{D}}{ }^{20}=-63.1$ (c 0.58 , acetone); ${ }^{1} \mathrm{H}$ NMR (400 MHz, $\left.\mathrm{CDCl}_{3}\right) \delta 7.85(\mathrm{~d}, J=7.6 \mathrm{~Hz}, 1 \mathrm{H}), 7.73(\mathrm{~s}, 1 \mathrm{H}), 7.28(\mathrm{~s}, 1 \mathrm{H})$, 7.25-7.19 (m, 5H), 7.19-7.09 (m, 4H), 6.96-6.90 (m, 1H), 6.82-6.76 (m, 2H), 6.74-6.70 (m, 1H), $6.60(\mathrm{~s}, 1 \mathrm{H}), 6.13-6.05(\mathrm{~m}, 2 \mathrm{H}), 5.93-5.89(\mathrm{~m}, 2 \mathrm{H}), 5.53(\mathrm{~d}, J=10.1 \mathrm{~Hz}, 1 \mathrm{H}), 4.00-3.91(\mathrm{~m}$, $1 \mathrm{H}), 3.90-3.83(\mathrm{~m}, 1 \mathrm{H}), 1.91(\mathrm{~s}, 3 \mathrm{H}) ;{ }^{13} \mathrm{C} \mathrm{NMR}\left(100 \mathrm{MHz}, \mathrm{CDCl}_{3}\right) \delta 149.8,147.0,141.7,139.9$, 137.2, 136.2, 132.4, 130.7, 130.0, 128.5, 127.2, 126.2, 126.1, 125.9, 122.9, 122.1, 120.2, 119.7, 116.7, 114.2, 111.2, 108.2, 101.0, 98.8, 77.3, 49.5, 44.9, 20.0; IR (KBr): 3702, 3690, 3676, 1751, $1522,1240,1153,743 \mathrm{~cm}^{-1} ;\left(\mathrm{C}_{33} \mathrm{H}_{27} \mathrm{NO}_{3}-\mathrm{H}\right)^{-}$requires $\mathrm{m} / z$ 484.1918, found $\mathrm{m} / z$ 484.1896; ee: $65 \%$, IB, hexane/isopropanol $=90 / 10, \mathrm{t}_{\mathrm{R}}=21.567$ (minor), $\mathrm{t}_{\mathrm{R}}=23.540$ (major).

(E)-2-(7-(2-chlorophenyl)-8-styryl-7,8-dihydro-6H-[1,3]dioxolo[4,5-g]chromen-6-yl)-1Hindole (3ha):

Yield: 62\% (31.7 mg); 91:9 dr; brown solid; m.p. $195.1-195.6{ }^{\circ} \mathrm{C} ;[\alpha]_{\mathrm{D}}{ }^{20}=-57.9$ (c 0.63 , acetone); ${ }^{1} \mathrm{H}$ NMR $\left(400 \mathrm{MHz} \mathrm{CDCl}_{3}\right) \delta 7.92(\mathrm{~s}, 1 \mathrm{H}), 7.79(\mathrm{~d}, J=7.0 \mathrm{~Hz}, 1 \mathrm{H}), 7.26-7.20(\mathrm{~m}$, $6 \mathrm{H}), 7.20-7.17(\mathrm{~m}, 1 \mathrm{H}), 7.16-7.10(\mathrm{~m}, 3 \mathrm{H}), 7.07(\mathrm{~m}, 2 \mathrm{H}), 6.94-6.90(\mathrm{~m}, 1 \mathrm{H}), 6.74(\mathrm{~s}, 1 \mathrm{H}), 6.55$ $(\mathrm{s}, 1 \mathrm{H}), 6.12-6.07(\mathrm{~m}, 2 \mathrm{H}), 5.90(\mathrm{~s}, 2 \mathrm{H}), 5.62(\mathrm{~d}, J=9.6 \mathrm{~Hz}, 1 \mathrm{H}), 4.38-4.27(\mathrm{~m}, 1 \mathrm{H}), 3.91-3.80$ $(\mathrm{m}, 1 \mathrm{H}) ;{ }^{13} \mathrm{C}$ NMR $\left(100 \mathrm{MHz}, \mathrm{CDCl}_{3}\right) \delta 149.7,147.1,141.8,139.4,137.2,136.1,134.8,132.6$, 130.6, 129.3, 128.5, 127.8, 127.4, 127.3, 127.1, 126.3, 123.1, 122.3, 119.9, 119.6, 116.2, 114.2, 111.1, 108.1, 101.1, 98.8, 75.3, 50.3, 44.7; IR (KBr): 3853, 3676, 3650, 3629, 1685, 1507, 1477, $1037,745 \mathrm{~cm}^{-1} ;\left(\mathrm{C}_{32} \mathrm{H}_{24} \mathrm{ClNO}_{3}-\mathrm{H}\right)^{-}$requires $\mathrm{m} / \mathrm{z} 504.1372$, found $\mathrm{m} / \mathrm{z}$ 504.1377; ee: $65 \%$, $\mathrm{IA}$, hexane/isopropanol $=70 / 30, \mathrm{t}_{\mathrm{R}}=10.020$ (minor), $\mathrm{t}_{\mathrm{R}}=27.087$ (major).

(E)-2-(7-(2-bromophenyl)-8-styryl-7,8-dihydro-6H-[1,3]dioxolo[4,5-g]chromen-6-yl)-1Hindole (3ia):

Yield: $96 \%$ (52.8 mg); 92:8 dr; brown solid; m.p. $185.0-185.4{ }^{\circ} \mathrm{C} ;[\alpha]_{\mathrm{D}}{ }^{20}=-59.3$ (c 1.06 , acetone); ${ }^{1} \mathrm{H} \mathrm{NMR}\left(400 \mathrm{MHz}, \mathrm{CDCl}_{3}\right) \delta 7.90(\mathrm{~s}, 1 \mathrm{H}), 7.81(\mathrm{~d}, J=7.3 \mathrm{~Hz}, 1 \mathrm{H}), 7.30-7.27(\mathrm{~m}$, $4 \mathrm{H}), 7.25-7.19(\mathrm{~m}, 4 \mathrm{H}), 7.18-7.10(\mathrm{~m}, 3 \mathrm{H}), 7.08(\mathrm{~d}, J=2.4 \mathrm{~Hz}, 1 \mathrm{H}), 6.88-6.82(\mathrm{~m}, 1 \mathrm{H}), 6.76$ (s, 
$1 \mathrm{H}), 6.56(\mathrm{~s}, 1 \mathrm{H}), 6.18-6.13(\mathrm{~m}, 1 \mathrm{H}), 6.12-6.05(\mathrm{~m}, 1 \mathrm{H}), 5.90(\mathrm{~s}, 2 \mathrm{H}), 5.63(\mathrm{~d}, J=10.3 \mathrm{~Hz}, 1 \mathrm{H})$, 4.37-4.27 (m, 1H), 3.91-3.82 (m, 1H); $\left.{ }^{13} \mathrm{C} \mathrm{NMR} \mathrm{(100} \mathrm{MHz,} \mathrm{CDCl}_{3}\right) \delta$ 149.7, 147.1, 141.9, 141.1, 137.2, 136.0, 132.7, 132.6, 130.5, 128.5, 128.1, 127.8, 127.3, 126.4, 126.1, 123.3, 122.3, 119.9, 119.7, 116.2, 114.0, 111.2, 108.1, 101.1, 98.8, 75.5, 50.7, 47.6; IR (KBr): 3418, 3055, 2885, 1499, $1477,1240,1152,1037,965,744 \mathrm{~cm}^{-1} ;\left(\mathrm{C}_{32} \mathrm{H}_{24} \mathrm{BrNO}_{3}-\mathrm{H}\right)^{-}$requires $m / z 548.0867$ found $m / z 548.0849$; ee: $66 \%$, IA, hexane/isopropanol $=70 / 30, t_{R}=10.603($ minor $), t_{R}=29.107$ (major).

(E)-2-(8-styryl-7-(m-tolyl)-7,8-dihydro-6H-[1,3]dioxolo[4,5-g]chromen-6-yl)-1H-indole (3ja): Yield: $84 \%$ (40.8 mg); 84:16 dr; brown solid; m.p. $148.3-148.6{ }^{\circ} \mathrm{C} ;[\alpha]_{\mathrm{D}}{ }^{20}=-29.2(\mathrm{c} 0.82$, acetone); ${ }^{1} \mathrm{H}$ NMR $\left(400 \mathrm{MHz}, \mathrm{CDCl}_{3}\right) \delta 7.82(\mathrm{~d}, J=7.4 \mathrm{~Hz}, 1 \mathrm{H}), 7.78(\mathrm{~s}, 1 \mathrm{H}), 7.26-7.18(\mathrm{~m}$, $6 \mathrm{H}), 7.17-7.11(\mathrm{~m}, 2 \mathrm{H}), 6.98-6.94(\mathrm{~m}, 1 \mathrm{H}), 6.85-6.77(\mathrm{~m}, 5 \mathrm{H}), 6.57(\mathrm{~s}, 1 \mathrm{H}), 6.20-6.16(\mathrm{~m}, 1 \mathrm{H})$, 6.06-5.98 (m, 1H), $5.91(\mathrm{~s}, 2 \mathrm{H}), 5.48(\mathrm{~d}, J=10.5 \mathrm{~Hz}, 1 \mathrm{H}), 4.00-3.93(\mathrm{~m}, 1 \mathrm{H}), 3.53-3.44(\mathrm{~m}$, 1H), $2.15(\mathrm{~s}, 3 \mathrm{H}) ;{ }^{13} \mathrm{C} \mathrm{NMR}\left(100 \mathrm{MHz}, \mathrm{CDCl}_{3}\right) \delta 149.8,146.9,141.7,140.9,137.5,137.2,136.2$, 132.7, 131.5, 129.0, 128.4, 128.0, 127.2, 126.2, 126.0, 125.4, 123.3, 122.1, 120.0, 119.7, 116.5, 114.4, 111.2, 108.2, 101.0, 98.8, 76.8, 50.4, 48.3, 21.4; IR (KBr): 3801, 3734, 3648, 3587, 1749, 1576, 1521, 1038, $746 \mathrm{~cm}^{-1} ;\left(\mathrm{C}_{33} \mathrm{H}_{27} \mathrm{NO}_{3}-\mathrm{H}\right)^{-}$requires $\mathrm{m} / \mathrm{z} 484.1918$, found $\mathrm{m} / \mathrm{z} 484.1905$; ee: $59 \%$, IA, hexane/isopropanol $=70 / 30, t_{R}=10.097$ (minor), $t_{R}=19.040$ (major).

(E)-2-(7-(3-chlorophenyl)-8-styryl-7,8-dihydro-6H-[1,3]dioxolo[4,5-g]chromen-6-yl)-1Hindole (3ka):

Yield: $98 \%$ (49.5 mg); 87:13 dr; brown solid; m.p. $160.3-160.9{ }^{\circ} \mathrm{C} ;[\alpha]_{\mathrm{D}}{ }^{20}=-30.5(\mathrm{c} 0.99$, acetone); ${ }^{1} \mathrm{H}$ NMR (400 MHz, $\left.\mathrm{CDCl}_{3}\right) \delta 7.87(\mathrm{~s}, 1 \mathrm{H}), 7.81(\mathrm{~d}, J=7.6 \mathrm{~Hz}, 1 \mathrm{H}), 7.30-7.27$ (m, $2 \mathrm{H}), 7.26-7.13(\mathrm{~m}, 6 \mathrm{H}), 7.04(\mathrm{~s}, 1 \mathrm{H}), 7.00-6.93(\mathrm{~m}, 2 \mathrm{H}), 6.84-6.80(\mathrm{~m}, 2 \mathrm{H}), 6.77(\mathrm{~s}, 1 \mathrm{H}), 6.56(\mathrm{~s}$, $1 \mathrm{H}), 6.22(\mathrm{~d}, J=15.8 \mathrm{~Hz}, 1 \mathrm{H}), 6.00(\mathrm{~m}, 1 \mathrm{H}), 5.91(\mathrm{~s}, 2 \mathrm{H}), 5.42(\mathrm{~d}, J=10.5 \mathrm{~Hz}, 1 \mathrm{H}), 3.98-3.92$ (m, 1H), 3.57-3.49 (m, 1H); $\left.{ }^{13} \mathrm{C} \mathrm{NMR} \mathrm{(100} \mathrm{MHz,} \mathrm{CDCl}_{3}\right) \delta 149.7,147.1,143.1,141.9,136.9$, 136.3, 133.9, 133.2, 130.8, 129.4, 128.5, 128.0, 127.4, 126.9, 126.7, 126.3, 125.7, 123.4, 122.3, $119.9,116.0,114.0,111.3,108.1,101.1,98.8,76.7,50.4,48.2$; IR (KBr): 3749, 3647, 3617, 3419, $1698,1683,1418,745,668 \mathrm{~cm}^{-1} ;\left(\mathrm{C}_{32} \mathrm{H}_{24} \mathrm{ClNO}_{3}-\mathrm{H}\right)^{-}$requires $\mathrm{m} / z 504.1372$, found $\mathrm{m} / z$ 504.1389; ee: $58 \%, I B$, hexane/isopropanol $=90 / 10, t_{R}=24.790$ (minor), $t_{R}=26.910$ (major).

(E)-2-(8-styryl-7-(p-tolyl)-7,8-dihydro-6H-[1,3]dioxolo[4,5-g]chromen-6-yl)-1H-indole (3la): Yield: 54\% (26.0 mg); 78:22 dr; brown solid; m.p. $220.1-221.9^{\circ} \mathrm{C} ;[\alpha]_{\mathrm{D}}{ }^{20}=-46.2(\mathrm{c} 0.52$, acetone); ${ }^{1} \mathrm{H}$ NMR $\left(400 \mathrm{MHz}, \mathrm{CDCl}_{3}\right) \delta 7.86-7.79(\mathrm{~m}, 2 \mathrm{H}), 7.28(\mathrm{~s}, 1 \mathrm{H}), 7.25-7.22(\mathrm{~m}, 4 \mathrm{H})$, 7.17-7.12 (m, 2H), 7.05-6.97 (m, 1H), $6.87(\mathrm{~s}, 4 \mathrm{H}), 6.79(\mathrm{~d}, J=2.4 \mathrm{~Hz}, 1 \mathrm{H}), 6.76(\mathrm{~s}, 1 \mathrm{H}), 6.56(\mathrm{~s}$, $1 \mathrm{H}), 6.20-6.16(\mathrm{~m}, 1 \mathrm{H}), 6.05-5.97(\mathrm{~m}, 1 \mathrm{H}), 5.90(\mathrm{~s}, 2 \mathrm{H}), 5.45(\mathrm{~d}, J=10.5 \mathrm{~Hz}, 1 \mathrm{H}), 3.97-3.92(\mathrm{~m}$, 1H), 3.54-3.47 (m, 1H), 2.16 (s, 3H); $\left.{ }^{13} \mathrm{C} \mathrm{NMR} \mathrm{(100} \mathrm{MHz,} \mathrm{CDCl}_{3}\right) \delta 149.8,147.0,141.7,137.9$, $137.2,136.3,135.8,132.7,131.5,128.9,128.5,128.1,127.2,126.3,126.0,123.4,122.2,120.2$, $119.8,116.5,114.5,111.2,108.3,101.0,98.8,77.0,50.0,48.3,21.0 ; \mathrm{IR}(\mathrm{KBr}): 3726,3675,3649$, $3587,1869,1670,1395,1152,744 \mathrm{~cm}^{-1} ;\left(\mathrm{C}_{33} \mathrm{H}_{27} \mathrm{NO}_{3}-\mathrm{H}\right)^{-}$requires $m / z 484.1918$, found $m / z$ 484.1902; ee: $85 \%$, IA, hexane/isopropanol $=70 / 30, \mathrm{t}_{\mathrm{R}}=10.557$ (minor), $\mathrm{t}_{\mathrm{R}}=18.093$ (major).

(E)-2-(7-(4-chlorophenyl)-8-styryl-7,8-dihydro-6H-[1,3]dioxolo[4,5-g]chromen-6-yl)-1Hindole (3ma):

Yield: 58\% (29.5 mg); 84:16 dr; brown solid; m.p. 85.2-86.0 ${ }^{\circ} \mathrm{C}$; $[\alpha]_{\mathrm{D}}{ }^{20}=-16.7$ (c 0.59 , acetone); ${ }^{1} \mathrm{H} \mathrm{NMR}\left(400 \mathrm{MHz}, \mathrm{CDCl}_{3}\right) \delta 7.88(\mathrm{~s}, 1 \mathrm{H}), 7.81(\mathrm{~d}, J=7.8 \mathrm{~Hz}, 1 \mathrm{H}), 7.30-7.26(\mathrm{~m}$, $2 \mathrm{H}), 7.26-7.25(\mathrm{~m}, 2 \mathrm{H}), 7.23-7.11(\mathrm{~m}, 4 \mathrm{H}), 7.06-7.00(\mathrm{~m}, 2 \mathrm{H}), 6.89(\mathrm{~d}, J=8.4 \mathrm{~Hz}, 2 \mathrm{H}), 6.79$ $(\mathrm{d}, J=2.5 \mathrm{~Hz}, 1 \mathrm{H}), 6.76(\mathrm{~s}, 1 \mathrm{H}), 6.55(\mathrm{~s}, 1 \mathrm{H}), 6.22-6.17(\mathrm{~m}, 1 \mathrm{H}), 6.04-5.95(\mathrm{~m}, 1 \mathrm{H}), 5.91(\mathrm{~s}$, 2H), $5.40(\mathrm{~d}, J=10.5 \mathrm{~Hz}, 1 \mathrm{H}), 3.96-3.90(\mathrm{~m}, 1 \mathrm{H}), 3.58-3.49(\mathrm{~m}, 1 \mathrm{H}) ;{ }^{13} \mathrm{C} \mathrm{NMR}(100 \mathrm{MHz}$, $\left.\mathrm{CDCl}_{3}\right) \delta 149.7,147.1,141.9,139.5,136.9,136.4,133.1,132.1,130.9,129.6,128.5,128.4,127.4$, 126.3, 125.7, 123.4, 122.4, 120.1, 120.0, 116.1, 114.1, 111.4, 108.2, 101.1, 98.8, 76.9, 50.0, 48.2; IR (KBr): 3870, 3711, 3690, 3629, 1844, 1684, 1576, 1395, $745 \mathrm{~cm}^{-1} ;\left(\mathrm{C}_{32} \mathrm{H}_{24} \mathrm{ClNO}_{3}-\mathrm{H}\right)^{-}$requires $m / z 504.1372$, found $m / z$ 504.1374; $е e: 97 \%$, IA, hexane/isopropanol $=70 / 30, t_{R}=11.823$ (minor), $\mathrm{t}_{\mathrm{R}}=20.797$ (major).

(E)-5-chloro-1-methyl-2-(7-phenyl-8-styryl-7,8-dihydro-6H-[1,3]dioxolo[4,5-g]chromen-6-yl)$1 H$-indole (3na): 
Yield:47\% (24.2 mg); 86:14 dr; brown solid; m.p. 205.1-205.7 ${ }^{\circ} \mathrm{C} ;[\alpha]_{\mathrm{D}}{ }^{20}=-11.4$ (c 0.48 , acetone); ${ }^{1} \mathrm{H}$ NMR (400 MHz, acetone- $\left.d_{6}\right) \delta 7.77(\mathrm{~m}, 1 \mathrm{H}), 7.30-7.23(\mathrm{~m}, 6 \mathrm{H}), 7.20-7.15(\mathrm{~m}$, $3 \mathrm{H}), 7.11-7.05(\mathrm{~m}, 4 \mathrm{H}), 6.99-6.94(\mathrm{~m}, 1 \mathrm{H}), 6.71(\mathrm{~s}, 1 \mathrm{H}), 6.43(\mathrm{~s}, 1 \mathrm{H}), 6.24-6.20(\mathrm{~m}, 1 \mathrm{H})$, 6.17-6.11 (m, 1H), $5.92(\mathrm{~d}, J=1.8 \mathrm{~Hz}, 2 \mathrm{H}), 5.57(\mathrm{~d}, J=10.6 \mathrm{~Hz}, 1 \mathrm{H}), 3.65(\mathrm{~s}, 3 \mathrm{H}), 3.62-3.56$ $(\mathrm{m}, 1 \mathrm{H}) ;{ }^{13} \mathrm{C}$ NMR $\left(100 \mathrm{MHz}\right.$, acetone- $\left.d_{6}\right) \delta 149.9,147.0,141.7,141.4,137.3,135.5,132.6$, 131.6, 130.3, 129.3, 128.6, 128.4, 128.0, 127.1, 126.2, 126.1, 124.4, 121.3, 119.1, 116.6, 113.1, $110.9,107.9,101.0,98.2,76.0,50.4,48.7,32.1$; IR (KBr): 3868 3852, 3688, 3627, 1791, 1733, $1698,1521,1507,746 \mathrm{~cm}^{-1} ;\left(\mathrm{C}_{33} \mathrm{H}_{26} \mathrm{ClNO}_{3}-\mathrm{H}\right)^{-}$requires $m / z$ 518.1528, found $m / z$ 518.1526; ee: $14 \%, I B$, hexane/isopropanol $=80 / 20, t_{R}=9.320$ (major), $t_{R}=9.850$ (minor).

(E)-2-(8-(2-methylstyryl)-7-phenyl-7,8-dihydro-6H-[1,3]dioxolo[4,5-g]chromen-6-yl)-1Hindole (3ab):

Yield: 98\% (47.5 mg); 88:12 dr; brown solid; m.p. $203.3-205.0{ }^{\circ} \mathrm{C} ;[\alpha]_{\mathrm{D}}{ }^{20}=-6.0(\mathrm{c} 0.95$, acetone); ${ }^{1} \mathrm{H} \mathrm{NMR}\left(400 \mathrm{MHz}, \mathrm{CDCl}_{3}\right) \delta 7.84(\mathrm{~d}, J=7.4 \mathrm{~Hz}, 1 \mathrm{H}), 7.77(\mathrm{~s}, 1 \mathrm{H}), 7.34-7.30$ (m, 1H), 7.21-7.15 (m, 3H), 7.14-7.09 (m, 4H), 7.09-7.04 (m, 2H), 7.03-7.00 (m, 2H), 6.82 (s, $1 \mathrm{H}), 6.78(\mathrm{~m}, 1 \mathrm{H}), 6.59(\mathrm{~s}, 1 \mathrm{H}), 6.33(\mathrm{~d}, J=15.5 \mathrm{~Hz}, 1 \mathrm{H}), 5.94-5.92(\mathrm{~m}, 2 \mathrm{H}), 5.88-5.80(\mathrm{~m}$, $1 \mathrm{H}), 5.52(\mathrm{~d}, J=10.5 \mathrm{~Hz}, 1 \mathrm{H}), 4.05-3.98(\mathrm{~m}, 1 \mathrm{H}), 3.60-3.49(\mathrm{~m}, 1 \mathrm{H}), 2.01(\mathrm{~s}, 3 \mathrm{H}) ;{ }^{13} \mathrm{C} \mathrm{NMR}$ $\left(100 \mathrm{MHz} \mathrm{CDCl}_{3}\right) \delta 149.7,147.0,141.7,141.0,136.6,136.2,135.2,132.7,131.5,130.0,128.4$, $128.2,127.2,126.4,126.1,126.0,123.4,122.2,120.1,119.8,116.5,114.4,111.2,108.1,101.0$, 98.8, 76.8, 50.4, 48.7, 19.5; IR (KBr): 3763, 3668, 3627, 2899, 1732, 1682, 1568, 1479, 1153, $744 \mathrm{~cm}^{-1}$; $\left(\mathrm{C}_{33} \mathrm{H}_{27} \mathrm{NO}_{3}-\mathrm{H}\right)^{-}$requires $\mathrm{m} / z$ 484.1918, found $m / z$ 484.1894; ee: $62 \%$, AD-H, hexane/isopropanol $=70 / 30, \mathrm{t}_{\mathrm{R}}=8.893$ (minor), $\mathrm{t}_{\mathrm{R}}=13.527$ (major).

(E)-2-(8-(3-methoxystyryl)-7-phenyl-7,8-dihydro-6H-[1,3]dioxolo[4,5-g]chromen-6-yl)-1Hindole (3ac):

Yield: 98\% (49.1 mg); 86:14 dr; brown solid; m.p. $120.9-121.2^{\circ} \mathrm{C} ;[\alpha]_{\mathrm{D}}{ }^{20}=-39.9$ (c 0.98 , acetone); ${ }^{1} \mathrm{H}$ NMR (400 MHz, $\left.\mathrm{CDCl}_{3}\right) \delta 7.84(\mathrm{~d}, J=7.3 \mathrm{~Hz}, 1 \mathrm{H}), 7.78(\mathrm{~s}, 1 \mathrm{H}), 7.21-7.16$ $(\mathrm{m}, 3 \mathrm{H}), 7.16-7.15(\mathrm{~m}, 1 \mathrm{H}), 7.11-7.05(\mathrm{~m}, 2 \mathrm{H}), 7.05-6.97(\mathrm{~m}, 3 \mathrm{H}), 6.84(\mathrm{~d}, J=7.8 \mathrm{~Hz}, 1 \mathrm{H})$, $6.78(\mathrm{~s}, 2 \mathrm{H}), 6.77-6.74(\mathrm{~m}, 2 \mathrm{H}), 6.58(\mathrm{~s}, 1 \mathrm{H}), 6.17-6.12(\mathrm{~m}, 1 \mathrm{H}), 6.07-5.99(\mathrm{~m}, 1 \mathrm{H}), 5.92-5.90$ $(\mathrm{m}, 2 \mathrm{H}), 5.48(\mathrm{~d}, J=10.5 \mathrm{~Hz}, 1 \mathrm{H}), 4.01-3.95(\mathrm{~m}, 1 \mathrm{H}), 3.78(\mathrm{~s}, 3 \mathrm{H}), 3.59-3.49(\mathrm{~m}, 1 \mathrm{H}) ;{ }^{13} \mathrm{C}$ NMR $\left(100 \mathrm{MHz}_{2} \mathrm{CDCl}_{3}\right) \delta 159.7,149.8,147.0,141.8,140.9,138.6,136.2,132.7,131.6,129.4$, $128.3,128.2,126.4,125.9,123.4,122.2,120.0,119.8,118.9,116.3,114.3,112.9,111.5,111.2$, 108.2, 101.0, 98.8, 76.9, 55.2, 50.5, 48.2; IR (KBr): 3904, 3690, 3675, 3421, 1734, 1670, 1522, $1153,746 \mathrm{~cm}^{-1} ;\left(\mathrm{C}_{33} \mathrm{H}_{27} \mathrm{NO}_{4}-\mathrm{H}\right)^{-}$requires $\mathrm{m} / z$ 500.1867, found $\mathrm{m} / z$ 500.1868; ee: $63 \%$, IA, hexane isopropanol $=70 / 30, \mathrm{t}_{\mathrm{R}}=11.337$ (minor), $\mathrm{t}_{\mathrm{R}}=26.440$ (major).

(E)-2-(8-(3-fluorostyryl)-7-phenyl-7,8-dihydro-6H-[1,3]dioxolo[4,5-g]chromen-6-yl)-1Hindole (3ad):

Yield: $98 \%$ (47.9 mg); 85:15 dr; brown solid m.p. $163.6-164.0{ }^{\circ} \mathrm{C} ;[\alpha]_{\mathrm{D}}{ }^{20}=-39.6(\mathrm{c} 0.96$, acetone); ${ }^{1} \mathrm{H} \mathrm{NMR}\left(400 \mathrm{MHz} \mathrm{CDCl}_{3}\right) \delta 7.83(\mathrm{~d}, J=7.3 \mathrm{~Hz}, 1 \mathrm{H}), 7.78(\mathrm{~s}, 1 \mathrm{H}), 7.23-7.13(\mathrm{~m}$, $5 \mathrm{H}), 7.08(\mathrm{~d}, J=7.5 \mathrm{~Hz}, 1 \mathrm{H}), 7.06-7.01(\mathrm{~m}, 1 \mathrm{H}), 7.01-6.96(\mathrm{~m}, 3 \mathrm{H}), 6.94-6.85(\mathrm{~m}, 2 \mathrm{H}), 6.77$ $(\mathrm{m}, 1 \mathrm{H}), 6.74(\mathrm{~s}, 1 \mathrm{H}), 6.58(\mathrm{~s}, 1 \mathrm{H}), 6.16-6.10(\mathrm{~m}, 1 \mathrm{H}), 6.08-5.99(\mathrm{~m}, 1 \mathrm{H}), 5.92(\mathrm{~s}, 2 \mathrm{H}), 5.48(\mathrm{~d}$, $J=10.5 \mathrm{~Hz}, 1 \mathrm{H}), 4.01-3.95(\mathrm{~m}, 1 \mathrm{H}), 3.58-3.49(\mathrm{~m}, 1 \mathrm{H}) ;{ }^{13} \mathrm{C} \mathrm{NMR}\left(100 \mathrm{MHz}, \mathrm{CDCl}_{3}\right) \delta 163.0$ $(\mathrm{d}, J=244.0 \mathrm{~Hz}), 149.8,147.1,141.8,140.8,139.4(\mathrm{~d}, J=7.0 \mathrm{~Hz}), 136.2,132.7,131.8,131.7$, $129.9(\mathrm{~d}, \mathrm{~J}=9.0 \mathrm{~Hz}), 128.3,128.2,126.5,125.9,123.4,122.2,122.1,119.9(\mathrm{~J}=21.0 \mathrm{~Hz}), 116.0$, $114.2,114.0(\mathrm{~d}, J=21.0 \mathrm{~Hz}), 112.6(\mathrm{~d}, J=22.0 \mathrm{~Hz}), 111.2,108.1,101.0,98.9,76.8,50.4,48.2$; IR (KBr): 3869, 3750, 3734, 3587, 1868, 1683, 1521, 1038, 748, $668 \mathrm{~cm}^{-1} ;\left(\mathrm{C}_{32} \mathrm{H}_{24} \mathrm{FNO}_{3}-\mathrm{H}\right)^{-}$ requires $m / z$ 488.1667, found $m / z$ 488.1675; ee: 60\%, AD-H, hexane/isopropanol = 70/30, $\mathrm{t}_{\mathrm{R}}=11.737$ (minor), $\mathrm{t}_{\mathrm{R}}=49.947$ (major).

(E)-2-(8-(4-methoxystyryl)-7-phenyl-7,8-dihydro-6H-[1,3]dioxolo[4,5-g]chromen-6-yl)-1Hindole (3ae):

Yield: $73 \%$ (36.5 mg); 89:11 dr; brown solid m.p. $207.6-207.7^{\circ} \mathrm{C} ;[\alpha]_{\mathrm{D}}{ }^{20}=-47.8(\mathrm{c} 0.73$, acetone); ${ }^{1} \mathrm{H}$ NMR $\left(400 \mathrm{MHz}\right.$, acetone- $\left.d_{6}\right) \delta 9.98(\mathrm{~s}, 1 \mathrm{H}), 7.81(\mathrm{~d}, J=7.7 \mathrm{~Hz}, 1 \mathrm{H}), 7.29(\mathrm{~d}, J=$ $8.0 \mathrm{~Hz}, 1 \mathrm{H}), 7.23-7.20(\mathrm{~m}, 2 \mathrm{H}), 7.17-7.13(\mathrm{~m}, 2 \mathrm{H}), 7.10(\mathrm{~m}, 1 \mathrm{H}), 7.06-7.00(\mathrm{~m}, 4 \mathrm{H}), 6.96-6.91$ $(\mathrm{m}, 1 \mathrm{H}), 6.82-6.78(\mathrm{~m}, 2 \mathrm{H}), 6.72(\mathrm{~s}, 1 \mathrm{H}), 6.43(\mathrm{~s}, 1 \mathrm{H}), 6.18-6.12(\mathrm{~m}, 1 \mathrm{H}), 6.01-5.94(\mathrm{~m}, 1 \mathrm{H})$, $5.91(\mathrm{~m}, 2 \mathrm{H}), 5.59(\mathrm{~d}, J=10.6 \mathrm{~Hz}, 1 \mathrm{H}), 4.01-3.94(\mathrm{~m}, 1 \mathrm{H}), 3.74(\mathrm{~s}, 3 \mathrm{H}), 3.67-3.60(\mathrm{~m}, 1 \mathrm{H})$; 
${ }^{13} \mathrm{C}$ NMR $\left(100 \mathrm{MHz}\right.$, acetone- $\left.d_{6}\right) \delta 159.2,150.1,147.0,141.8,141.5,136.7,132.0,130.0,129.4$, $128.6,127.8,127.3,126.5,126.0,124.4,121.3,119.7,118.9,116.9,114.1,113.8,111.4,108.0$, 101.0, 98.2, 76.6, 54.6, 50.4, 48.7; IR (KBr): 3742, 3720, 3708, 3306, 1574, 1434, 1247, 745, $668 \mathrm{~cm}^{-1} ;\left(\mathrm{C}_{33} \mathrm{H}_{27} \mathrm{NO}_{4}-\mathrm{H}\right)^{-}$requires $m / z$ 500.1867, found $m / z$ 500.1871; ee: $77 \%$, AD-H, hexane/isopropanol $=80 / 20, \mathrm{t}_{\mathrm{R}}=22.810$ (minor), $\mathrm{t}_{\mathrm{R}}=55.720$ (major).

(E)-2-(8-(4-fluorostyryl)-7-phenyl-7,8-dihydro-6H-[1,3]dioxolo[4,5-g]chromen-6-yl)-1Hindole (3af):

Yield: 53\% (25.9 mg); 75:25 dr; brown solid; m.p. 81.4-82.6 ${ }^{\circ} \mathrm{C} ;[\alpha]_{\mathrm{D}}{ }^{20}=-53.3(\mathrm{c} 0.52$, acetone); ${ }^{1} \mathrm{H}$ NMR $\left(400 \mathrm{MHz}, \mathrm{CDCl}_{3}\right) \delta 7.85-7.79(\mathrm{~m}, 2 \mathrm{H}), 7.23(\mathrm{~d}, J=7.7 \mathrm{~Hz}, 1 \mathrm{H}), 7.19-7.12$ $(\mathrm{m}, 6 \mathrm{H}), 7.07(\mathrm{~d}, J=7.5 \mathrm{~Hz}, 1 \mathrm{H}), 7.04-7.01(\mathrm{~m}, 1 \mathrm{H}), 6.99-6.96(\mathrm{~m}, 2 \mathrm{H}), 6.95-6.91(\mathrm{~m}, 2 \mathrm{H}), 6.78$ $(\mathrm{m}, 1 \mathrm{H}), 6.75(\mathrm{~s}, 1 \mathrm{H}), 6.56(\mathrm{~s}, 1 \mathrm{H}), 6.13-6.09(\mathrm{~m}, 1 \mathrm{H}), 5.91(\mathrm{~s}, 2 \mathrm{H}), 5.47(\mathrm{~d}, J=10.5 \mathrm{~Hz}, 1 \mathrm{H})$, 3.98-3.93 (m, 1H), 3.56-3.49 (m, 1H); $\left.{ }^{13} \mathrm{C} \mathrm{NMR} \mathrm{(100} \mathrm{MHz,} \mathrm{CDCl}_{3}\right) \delta 162.1$ (d, J = 245.0 Hz), 149.8, 147.0, 141.7, 140.9, 136.2, 133.3, 133.2, 131.6, $131.1(\mathrm{~d}, J=2.0 \mathrm{~Hz}) 129.1,128.2(\mathrm{~d}$, $J=13.0 \mathrm{~Hz}), 127.7(\mathrm{~d}, J=8.0 \mathrm{~Hz}), 126.4,125.9,123.3,122.2,120.0,119.8,116.3,115.3(\mathrm{~d}$, $J=21.0 \mathrm{~Hz}), 114.3,111.2,108.1,101.0,98.8,76.9,50.5,48.2 ; \mathrm{IR}(\mathrm{KBr}): 3891,3884,3734,3669$, $3446,2970,1669,1066,749,668 \mathrm{~cm}^{-1} ;\left(\mathrm{C}_{32} \mathrm{H}_{24} \mathrm{FNO}_{3}-\mathrm{H}\right)^{-}$requires $\mathrm{m} / z$ 488.1667, found $\mathrm{m} / \mathrm{z}$ 488.1668; ee: 97\%, AD-H, hexane/isopropanol $=70 / 30, t_{R}=15.207$ (minor), $t_{R}=22.800$ (major).

(E)-2-(8-(4-chlorostyryl)-7-phenyl-7,8-dihydro-6H-[1,3]dioxolo[4,5-g]chromen-6-yl)-1Hindole (3ag):

Yield: 57\% (28.7 mg); 84:16 dr; brown solid; m.p. $175.7-176.2{ }^{\circ} \mathrm{C}$; $[\alpha]_{\mathrm{D}}{ }^{20}=-89.5$ (c 0.51 , acetone); ${ }^{1} \mathrm{H}$ NMR (400 MHz, $\left.\mathrm{CDCl}_{3}\right) \delta 7.85-7.78(\mathrm{~m}, 2 \mathrm{H}), 7.25-7.21(\mathrm{~m}, 2 \mathrm{H}), 7.19(\mathrm{~s}, 1 \mathrm{H})$, 7.17-7.15 (m, 1H), 7.14-7.13 (m, 2H), 7.12-7.11 (m, 1H), 7.10-7.05 (m, 2H), 7.04-7.00 (m, $1 \mathrm{H}), 6.99-6.95(\mathrm{~m}, 2 \mathrm{H}), 6.78(\mathrm{~d}, J=2.5 \mathrm{~Hz}, 1 \mathrm{H}), 6.73(\mathrm{~s}, 1 \mathrm{H}), 6.57(\mathrm{~s}, 1 \mathrm{H}), 6.12-6.06(\mathrm{~m}, 1 \mathrm{H})$, 6.02-5.94 (m, 1H), 5.92-5.89 (m, 2H), $5.47(\mathrm{~d}, J=10.5 \mathrm{~Hz}, 1 \mathrm{H}), 3.99-3.93(\mathrm{~m}, 1 \mathrm{H}), 3.57-3.47$ $(\mathrm{m}, 1 \mathrm{H}) ;{ }^{13} \mathrm{C} \mathrm{NMR}\left(100 \mathrm{MHz}, \mathrm{CDCl}_{3}\right)$ 149.8, 147.0, 141.8, 140.9, 136.2, 135.5, 132.8, 132.0, $131.6,128.5,128.3,128.2,127.4,126.5,125.9,123.3,122.2,120.0,119.8,116.1,114.3,111.2$, 108.1, 101.0, 98.8, 76.8, 50.5, 48.2; IR (KBr): 3869, 3688, 3627, 1791, 1683, 1521, 1076, 749, $668 \mathrm{~cm}^{-1} ;\left(\mathrm{C}_{32} \mathrm{H}_{24} \mathrm{ClNO}_{3}-\mathrm{H}\right)^{-}$requires $\mathrm{m} / z$ 504.1372, found $\mathrm{m} / z$ 504.1379; ee: 98\%, IB, hexane/isopropanol $=70 / 30, \mathrm{t}_{\mathrm{R}}=7.900$ (major), $\mathrm{t}_{\mathrm{R}}=8.993$ (minor).

(E)-2-(7-phenyl-8-(2-(thiophen-2-yl)vinyl)-7,8-dihydro-6H-[1,3]dioxolo[4,5-g]chromen-6-yl)$1 H$-indole (3ah):

Yield: $70 \%$ (33.2 mg); 85:15 dr; brown solid; m.p. $216.2-218.0{ }^{\circ} \mathrm{C} ;[\alpha]_{\mathrm{D}}{ }^{20}=-53.9(\mathrm{c} 0.66$, acetone); ${ }^{1} \mathrm{H}$ NMR $\left(400 \mathrm{MHz}, \mathrm{CDCl}_{3}\right) \delta 7.84-7.79(\mathrm{~m}, 2 \mathrm{H}), 7.25-7.21(\mathrm{~m}, 1 \mathrm{H}), 7.15-7.11(\mathrm{~m}$, $2 \mathrm{H}), 7.10-7.06(\mathrm{~m}, 3 \mathrm{H}), 7.04-7.01(\mathrm{~m}, 1 \mathrm{H}), 7.00-6.97(\mathrm{~m}, 2 \mathrm{H}), 6.91-6.87(\mathrm{~m}, 1 \mathrm{H}), 6.79-6.75$ $(\mathrm{m}, 3 \mathrm{H}), 6.55(\mathrm{~s}, 1 \mathrm{H}), 6.28(\mathrm{~d}, J=15.6 \mathrm{~Hz}, 1 \mathrm{H}), 5.92-5.90(\mathrm{~m}, 2 \mathrm{H}), 5.89-5.81(\mathrm{~m}, 1 \mathrm{H}), 5.46$ $(\mathrm{d}, J=10.5 \mathrm{~Hz}, 1 \mathrm{H}), 3.95-3.89(\mathrm{~m}, 1 \mathrm{H}), 3.55-3.47(\mathrm{~m}, 1 \mathrm{H}) ;{ }^{13} \mathrm{C} \mathrm{NMR}\left(100 \mathrm{MHz}, \mathrm{CDCl}_{3}\right) \delta$ $149.8,147.1,142.2,141.8,141.0,136.3,131.1,128.3,128.2,127.2,126.5,126.0,125.9,125.1$, 123.7, 123.4, 122.2, 120.1, 119.8, 116.2, 114.3, 111.2, 108.2, 101.1, 98.9, 76.9, 50.5, 48.2; IR (KBr): $3868,3851,3742,3674,3565,1715,1506,1265,1153,743 \mathrm{~cm}^{-1} ;\left(\mathrm{C}_{30} \mathrm{H}_{23} \mathrm{NO}_{3} \mathrm{~S}-\mathrm{H}\right)^{-}$requires $m / z 476.1326$, found $m / z$ 476.1315; ee: $87 \%$, IB, hexane/isopropanol $=90 / 10, t_{R}=22.563$ (minor), $\mathrm{t}_{\mathrm{R}}=24.167$ (major).

(E)-3-(7-(4-chlorophenyl)-8-styryl-7,8-dihydro-6H-[1,3]dioxolo[4,5-g]chromen-6-yl)-1-methyl1H-indole:(3oa)

Yield: $53 \%$ (27.7 mg); $91: 9 \mathrm{dr}$; white solid; m.p. $183.0-183.7{ }^{\circ} \mathrm{C} ;[\alpha]_{\mathrm{D}}{ }^{20}=-46.6(\mathrm{c} 0.15$, acetone); ${ }^{1} \mathrm{H} \mathrm{NMR}\left(400 \mathrm{MHz}, \mathrm{CDCl}_{3}\right) \delta 7.75(\mathrm{~d}, J=7.9 \mathrm{~Hz}, 1 \mathrm{H}), 7.25-7.17(\mathrm{~m}, 7 \mathrm{H}), 7.13-7.08$ $(\mathrm{m}, 1 \mathrm{H}), 7.03(\mathrm{~d}, J=8.2 \mathrm{~Hz}, 2 \mathrm{H}), 6.91(\mathrm{~d}, J=8.3 \mathrm{~Hz}, 2 \mathrm{H}), 6.73(\mathrm{~d}, J=4.3 \mathrm{~Hz}, 2 \mathrm{H}), 6.52(\mathrm{~s}$, $1 \mathrm{H}), 6.15(\mathrm{~d}, J=15.7 \mathrm{~Hz}, 1 \mathrm{H}), 6.01-5.94(\mathrm{~m}, 1 \mathrm{H}), 5.90(\mathrm{~s}, 2 \mathrm{H}), 5.40(\mathrm{~d}, J=10.5 \mathrm{~Hz}, 1 \mathrm{H})$, 3.92-3.86 (m, 1H), $3.62(\mathrm{~s}, 3 \mathrm{H}), 3.56-3.49(\mathrm{~m}, 1 \mathrm{H}) ;{ }^{13} \mathrm{C} \mathrm{NMR}\left(100 \mathrm{MHz}, \mathrm{CDCl}_{3}\right) \delta$ 149.7, 147.0, $141.8,139.6,137.2$, 136.9, 133.0, 132.0, 130.9, 129.5, 128.5, 128.3, 128.0, 127.4, 126.3, 126.2, $121.8,119.9,119.4,116.0,112.4,109.4,108.1,101.0,98.8,76.5,50.0,48.5,32.7$; IR (KBr): 3852, $3749,3648,2920,1477,1238,1152,1037,1013,742 \mathrm{~cm}^{-1} ;\left(\mathrm{C}_{33} \mathrm{H}_{26} \mathrm{ClNO}_{3}+\mathrm{H}\right)^{+}$requires $m / z$ 
520.1674, found $m / z$ 520.1668; ee: $83 \%$, AD-H, hexane $/$ isopropanol $=70 / 30, t_{R}=9.900$ (minor), $\mathrm{t}_{\mathrm{R}}=10.893$ (major).

Procedure for one-mmol-scale synthesis of product 3aa

To the mixture of 3-vinylindole $\mathbf{1 a}(1.0 \mathrm{mmol})$, ortho-quinone methide $\mathbf{2 a}(1.2 \mathrm{mmol})$, catalyst (R)-4c $(0.1 \mathrm{mmol})$, and $\mathrm{MgSO}_{4}(500 \mathrm{mg})$ was added toluene $(10 \mathrm{~mL})$. Then, the reaction mixture was stirred at $0{ }^{\circ} \mathrm{C}$ for $12 \mathrm{~h}$. After completion of the reaction, which was indicated by TLC, the reaction mixture was directly purified through flash column chromatography to afford product 3aa in 88\% yield (414 mg) with 86:14 $\mathrm{dr}$ and $64 \%$ ee.

\section{General procedure for the synthesis of products 6}

To the mixture of 3-vinylindoles $1(0.1 \mathrm{mmol})$, ortho-hydroxybenzyl alcohols 5 (0.12 $\mathrm{mmol})$, catalyst $4 \mathbf{e}(0.01 \mathrm{mmol})$ were added toluene $(1 \mathrm{~mL})$. Then, the reaction mixture was stirred at $0{ }^{\circ} \mathrm{C}$ for $6 \mathrm{~h}$. After completion of the reaction, which was indicated by TLC, the reaction mixture was directly purified through flash column chromatography to afford products 6 .

\section{(E)-3-(3,4-diphenylchroman-2-yl)-1H-indole (6aa):}

Yield: 61\% (24.5 mg); 77:23 dr; white solid; m.p. 95.0-97.0 ${ }^{\circ} \mathrm{C} ;[\alpha]_{\mathrm{D}}{ }^{20}=-169.4$ (c 0.26 , acetone); ${ }^{1} \mathrm{H}$ NMR (400 MHz, $\left.\mathrm{CDCl}_{3}\right) \delta 7.84(\mathrm{~s}, 1 \mathrm{H}), 7.79(\mathrm{~d}, J=7.7 \mathrm{~Hz}, 1 \mathrm{H}), 7.25-7.20(\mathrm{~m}$, 2H), 7.17-7.08 (m, 5H), 7.07-7.01 (m, 2H), 7.00-6.97 (m, 1H), 6.97-6.92 (m, 2H), 6.91-6.86 $(\mathrm{m}, 2 \mathrm{H}), 6.72(\mathrm{~d}, J=7.5 \mathrm{~Hz}, 2 \mathrm{H}), 6.59(\mathrm{~d}, J=7.5 \mathrm{~Hz}, 2 \mathrm{H}), 5.93(\mathrm{~d}, J=10.7 \mathrm{~Hz}, 1 \mathrm{H}), 4.40(\mathrm{~d}$, $J=5.2 \mathrm{~Hz}, 1 \mathrm{H}), 4.20-4.11(\mathrm{~m}, 1 \mathrm{H}) ;{ }^{13} \mathrm{C} \mathrm{NMR}\left(100 \mathrm{MHz}, \mathrm{CDCl}_{3}\right) \delta 154.9,141.5,139.6,136.2$, $130.6,130.2,129.1,128.1,127.5,126.4,126.2,126.1,124.8,123.7,122.2,120.4,119.9,119.8$, 116.8, 114.7, 111.2, 71.2, 48.7, 48.1; IR (KBr): 3648, 3566, 1733, 1456, 1418, 1242, 1111, 990, 748, $700 \mathrm{~cm}^{-1} ;\left(\mathrm{C}_{29} \mathrm{H}_{23} \mathrm{NO}+\mathrm{H}\right)^{+}$requires $\mathrm{m} / \mathrm{z} 402.1853$, found $\mathrm{m} / \mathrm{z}$ 402.1853; ee: $79 \%$, OD-H, hexane $/$ isopropanol $=95 / 5, t_{R}=20.910$ (major), $t_{R}=23.560$ (minor).

(E)-3-(3,4-diphenylchroman-2-yl)-5-methyl-1H-indole (6ba):

Yield: 50\% (20.7 mg); 75:25 dr; white solid; m.p. 84.0-86.0 ${ }^{\circ} \mathrm{C} ;[\alpha]_{\mathrm{D}}{ }^{20}=-145.2$ (c 0.17 , acetone); ${ }^{1} \mathrm{H} \mathrm{NMR}\left(400 \mathrm{MHz}, \mathrm{CDCl}_{3}\right) \delta 7.76(\mathrm{~s}, 1 \mathrm{H}), 7.56(\mathrm{~s}, 1 \mathrm{H}), 7.25-7.20(\mathrm{~m}, 1 \mathrm{H}), 7.18-7.09$ $(\mathrm{m}, 4 \mathrm{H}), 7.08-7.04(\mathrm{~m}, 1 \mathrm{H}), 7.04-7.01(\mathrm{~m}, 1 \mathrm{H}), 7.00-6.91(\mathrm{~m}, 4 \mathrm{H}), 6.91-6.86(\mathrm{~m}, 1 \mathrm{H}), 6.84(\mathrm{~d}$, $J=2.2 \mathrm{~Hz}, 1 \mathrm{H}), 6.72(\mathrm{~d}, J=7.3 \mathrm{~Hz}, 2 \mathrm{H}), 6.60(\mathrm{~d}, J=7.4 \mathrm{~Hz}, 2 \mathrm{H}), 5.90(\mathrm{~d}, J=10.6 \mathrm{~Hz}, 1 \mathrm{H}), 4.40$ $(\mathrm{d}, J=5.3 \mathrm{~Hz}, 1 \mathrm{H}), 4.20-4.11(\mathrm{~m}, 1 \mathrm{H}), 2.44(\mathrm{~s}, 3 \mathrm{H}) ;{ }^{13} \mathrm{C} \mathrm{NMR}\left(100 \mathrm{MHz}, \mathrm{CDCl}_{3}\right) \delta 154.9,141.6$, $139.7,134.6,130.6,130.3,129.1,129.0,128.1,127.5,127.4,126.4,126.2,124.8,123.8,120.3$, $119.4,116.9,114.1,110.8,71.2,48.7,47.9,21.6$; IR (KBr): 3648, 3566, 2922, 1748, 1558, 1507, $1456,1242,750,668 \mathrm{~cm}^{-1} ;\left(\mathrm{C}_{30} \mathrm{H}_{25} \mathrm{NO}+\mathrm{H}\right)^{+}$requires $\mathrm{m} / z$ 416.2009, found $\mathrm{m} / \mathrm{z}$ 416.1992; ee: $76 \%, \mathrm{AD}-\mathrm{H}$, hexane/isopropanol $=70 / 30, \mathrm{t}_{\mathrm{R}}=5.200$ (minor), $\mathrm{t}_{\mathrm{R}}=7.937$ (major).

\section{(E)-6-chloro-3-(3,4-diphenylchroman-2-yl)-1H-indole (6pa):}

Yield: $72 \%$ (31.3 mg); 75:25 dr; white solid; m.p. $112.0-114.0{ }^{\circ} \mathrm{C} ;[\alpha]_{\mathrm{D}}{ }^{20}=-253.5$ (c 0.20 , acetone); ${ }^{1} \mathrm{H}$ NMR (400 MHz, $\left.\mathrm{CDCl}_{3}\right) \delta 7.77(\mathrm{~s}, 1 \mathrm{H}), 7.69(\mathrm{~d}, J=8.5 \mathrm{~Hz}, 1 \mathrm{H}), 7.22(\mathrm{~d}$, $J=7.7 \mathrm{~Hz}, 1 \mathrm{H}), 7.20-7.10(\mathrm{~m}, 4 \mathrm{H}), 7.09-7.01(\mathrm{~m}, 3 \mathrm{H}), 7.01-6.97(\mathrm{~m}, 1 \mathrm{H}), 6.97-6.87(\mathrm{~m}, 3 \mathrm{H})$, 6.86-6.83 (m, 1H), $6.72(\mathrm{~d}, J=7.4 \mathrm{~Hz}, 2 \mathrm{H}), 6.55(\mathrm{~d}, J=7.4 \mathrm{~Hz}, 2 \mathrm{H}), 5.87(\mathrm{~d}, J=10.9 \mathrm{~Hz}, 1 \mathrm{H})$, $4.39(\mathrm{~d}, J=5.2 \mathrm{~Hz}, 1 \mathrm{H}), 4.14-4.06(\mathrm{~m}, 1 \mathrm{H}) ;{ }^{13} \mathrm{C} \mathrm{NMR}\left(100 \mathrm{MHz}, \mathrm{CDCl}_{3}\right) \delta 154.7,141.4,139.3$, $136.5,130.6,130.3,129.1,128.2,128.1,127.5,126.5,126.4,124.8,124.6,124.3,120.7,120.6$, 116.8, 114.9, 111.1, 71.0, 48.7, 48.3; IR (KBr): 3750, 3724, 3648, 3026, 1488, 1456, 1239, 1216, $752,705 \mathrm{~cm}^{-1} ;\left(\mathrm{C}_{29} \mathrm{H}_{22} \mathrm{ClNO}+\mathrm{H}\right)^{+}$requires $\mathrm{m} / z$ 436.1463, found $\mathrm{m} / \mathrm{z}$ 436.1460; ee: $74 \%$, $\mathrm{AD}-\mathrm{H}$, hexane/isopropanol $=70 / 30, \mathrm{t}_{\mathrm{R}}=5.843$ (minor), $\mathrm{t}_{\mathrm{R}}=7.477$ (major).

\section{(E)-6-bromo-3-(3,4-diphenylchroman-2-yl)-1H-indole (6da):}

Yield: 77\% (36.8 mg); 78:22 dr; white solid; m.p. 95.0-97.0 ${ }^{\circ} \mathrm{C} ;[\alpha]_{\mathrm{D}}{ }^{20}=-203.3$ (c 0.28 , acetone); ${ }^{1} \mathrm{H}$ NMR (400 MHz, $\left.\mathrm{CDCl}_{3}\right) \delta 7.77(\mathrm{~s}, 1 \mathrm{H}), 7.64(\mathrm{~d}, J=8.5 \mathrm{~Hz}, 1 \mathrm{H}), 7.33(\mathrm{~s}, 1 \mathrm{H})$, 7.24-7.15 (m, 3H), 7.15-7.10 (m, 2H), 7.05-7.01 (m, 2H), $6.99(\mathrm{~d}, J=7.3 \mathrm{~Hz}, 1 \mathrm{H}), 6.97-6.92(\mathrm{~m}$, $2 \mathrm{H}), 6.92-6.87(\mathrm{~m}, 1 \mathrm{H}), 6.82(\mathrm{~d}, J=2.1 \mathrm{~Hz}, 1 \mathrm{H}), 6.72(\mathrm{~d}, J=7.4 \mathrm{~Hz}, 2 \mathrm{H}), 6.55(\mathrm{~d}, J=7.4 \mathrm{~Hz}$, 2H), $5.87(\mathrm{~d}, J=10.9 \mathrm{~Hz}, 1 \mathrm{H}), 4.39(\mathrm{~d}, J=5.2 \mathrm{~Hz}, 1 \mathrm{H}), 4.13-4.06(\mathrm{~m}, 1 \mathrm{H}) ;{ }^{13} \mathrm{C} \mathrm{NMR}(100 \mathrm{MHz}$, $\left.\mathrm{CDCl}_{3}\right) \delta 154.7,141.4,139.3,137.0,130.6,130.3,129.1,128.2,127.5,126.5,126.4,124.9,124.8$, 124.3, 123.1, 121.1, 120.6, 116.7, 115.7, 114.9, 114.1, 71.0, 48.7, 48.3; IR (KBr): 3648, 3566, 1716, $1540,1507,1456,1226,801,750,668 \mathrm{~cm}^{-1} ;\left(\mathrm{C}_{29} \mathrm{H}_{22} \mathrm{BrNO}+\mathrm{H}\right)^{+}$requires $\mathrm{m} / z 480.0958$, found 
$m / z$ 480.0947; ee: 74\%, AD-H, hexane/isopropanol $=70 / 30, t_{R}=6.190($ minor $), t_{R}=8.233$ (major).

\section{(E)-3-(3,4-diphenylchroman-2-yl)-7-methyl-1H-indole (6ea):}

Yield: 53\% (21.9 mg); 75:25 dr; white solid; m.p. 92.0-94.0 ${ }^{\circ} \mathrm{C} ;[\alpha]_{\mathrm{D}}{ }^{20}=-158.6(\mathrm{c} 0.19$, acetone); ${ }^{1} \mathrm{H}$ NMR (400 MHz, $\left.\mathrm{CDCl}_{3}\right) \delta 7.72(\mathrm{~s}, 1 \mathrm{H}), 7.63(\mathrm{~d}, J=7.9 \mathrm{~Hz}, 1 \mathrm{H}), 7.25-7.19(\mathrm{~m}$, $1 \mathrm{H}), 7.18-7.09(\mathrm{~m}, 3 \mathrm{H}), 7.06-6.98(\mathrm{~m}, 4 \mathrm{H}), 6.97-6.92(\mathrm{~m}, 3 \mathrm{H}), 6.91-6.85(\mathrm{~m}, 2 \mathrm{H}), 6.72(\mathrm{~d}$, $J=7.4 \mathrm{~Hz}, 2 \mathrm{H}), 6.61(\mathrm{~d}, J=7.4 \mathrm{~Hz}, 2 \mathrm{H}), 5.92(\mathrm{~d}, J=10.6 \mathrm{~Hz}, 1 \mathrm{H}), 4.39(\mathrm{~d}, J=5.2 \mathrm{~Hz}, 1 \mathrm{H})$, 4.21-4.11 (m, 1H), 2.33 (s, 3H); $\left.{ }^{13} \mathrm{C} \mathrm{NMR} \mathrm{(100} \mathrm{MHz,} \mathrm{CDCl}_{3}\right) \delta 154.9,141.5,139.7,135.8$, $130.6,130.2,129.1,128.1,127.5,127.4,126.4,126.2,125.6,124.8,123.4,122.7,120.3,120.0$, $117.5,116.8,115.2,71.2,48.7,48.0,16.5$; IR (KBr): 3628, 3587, 3566, 3030, 1868, 1716, 1569, $1558,749,668 \mathrm{~cm}^{-1} ;\left(\mathrm{C}_{30} \mathrm{H}_{25} \mathrm{NO}+\mathrm{H}\right)^{+}$requires $\mathrm{m} / z$ 416.2009, found $\mathrm{m} / z$ 416.2004; ee: $74 \%$, $\mathrm{AD}-\mathrm{H}$, hexane/isopropanol $=70 / 30, \mathrm{t}_{\mathrm{R}}=5.437$ (major), $\mathrm{t}_{\mathrm{R}}=6.597$ (minor).

(E)-7-chloro-3-(3,4-diphenylchroman-2-yl)-1H-indole (6qa):

Yield: 90\% (39.2 mg); 82:18 dr; white solid; m.p. $55.0-57.0{ }^{\circ} \mathrm{C}$; $[\alpha]_{\mathrm{D}}{ }^{20}=-191.1$ (c 0.54 , acetone); ${ }^{1} \mathrm{H} \mathrm{NMR}\left(400 \mathrm{MHz} \mathrm{CDCl}_{3}\right) \delta 8.07(\mathrm{~s}, 1 \mathrm{H}), 7.70(\mathrm{~d}, J=7.9 \mathrm{~Hz}, 1 \mathrm{H}), 7.25-7.21(\mathrm{~m}$, 1H), 7.20-7.11 (m, 4H), 7.08-6.99 (m, 4H), $6.99-6.88(\mathrm{~m}, 4 \mathrm{H}), 6.73(\mathrm{~d}, J=7.2 \mathrm{~Hz}, 2 \mathrm{H}), 6.58$ $(\mathrm{d}, J=7.3 \mathrm{~Hz}, 2 \mathrm{H}), 5.91(\mathrm{~d}, J=10.9 \mathrm{~Hz}, 1 \mathrm{H}), 4.40(\mathrm{~d}, J=5.1 \mathrm{~Hz}, 1 \mathrm{H}), 4.20-4.07(\mathrm{~m}, 1 \mathrm{H})$; ${ }^{13} \mathrm{C}$ NMR $\left(100 \mathrm{MHz}, \mathrm{CDCl}_{3}\right) \delta 154.7,141.4,139.3,133.5,130.6,130.3,129.1,128.2,127.6$, $127.5,126.5,126.4,124.8,124.3,121.6,120.7,120.6,118.6,116.8,116.7,115.9,71.1,48.8,48.2$; IR (KBr): 3648, 3628, 3566, 3420, 1507, 1472, 1339, 1241, 752, $700 \mathrm{~cm}^{-1} ;\left(\mathrm{C}_{29} \mathrm{H}_{22} \mathrm{ClNO}+\mathrm{H}\right)^{+}$ requires $m / z$ 436.1463, found $m / z$ 436.1459; ee: 75\%, AD-H, hexane/isopropanol = 70/30, $\mathrm{t}_{\mathrm{R}}=5.490$ (major), $\mathrm{t}_{\mathrm{R}}=6.753$ (minor).

(E)-7-bromo-3-(3,4-diphenylchroman-2-yl)-1H-indole (6ra):

Yield: 89\% (42.7 mg); 83:17 dr; white solid; m.p. 87.0-89.0 ${ }^{\circ} \mathrm{C} ;[\alpha]_{\mathrm{D}}{ }^{20}=-194.8$ (c 0.56 , acetone); ${ }^{1} \mathrm{H} \mathrm{NMR}\left(400 \mathrm{MHz}, \mathrm{CDCl}_{3}\right) \delta 8.04(\mathrm{~s}, 1 \mathrm{H}), 7.75(\mathrm{~d}, J=8.0 \mathrm{~Hz}, 1 \mathrm{H}), 7.30(\mathrm{~d}, J=7.6$ $\mathrm{Hz}, 1 \mathrm{H}), 7.23(\mathrm{~d}, J=7.5 \mathrm{~Hz}, 1 \mathrm{H}), 7.20-7.12(\mathrm{~m}, 3 \mathrm{H}), 7.07-6.94(\mathrm{~m}, 7 \mathrm{H}), 6.93-6.88(\mathrm{~m}, 1 \mathrm{H})$, $6.73(\mathrm{~d}, J=7.3 \mathrm{~Hz}, 2 \mathrm{H}), 6.58(\mathrm{~d}, J=7.4 \mathrm{~Hz}, 2 \mathrm{H}), 5.90(\mathrm{~d}, J=10.9 \mathrm{~Hz}, 1 \mathrm{H}), 4.40(\mathrm{~d}, J=5.2 \mathrm{~Hz}$, 1H), 4.20-4.08 (m, 1H); ${ }^{13} \mathrm{C}$ NMR (100 MHz, $\left.\mathrm{CDCl}_{3}\right) \delta 154.7,141.4,139.3,134.9,130.6,130.3$, 129.1, 128.2, 127.6, 127.5, 127.3, 126.5, 126.4, 124.8, 124.6, 124.3, 121.0, 120.6, 119.2, 116.8, 116.0, 104.8, 71.1, 48.8, 48.2; IR (KBr): 3648, 3628, 3566, 1868, 1716, 1507, 1456, 1339, 751, $668 \mathrm{~cm}^{-1} ;\left(\mathrm{C}_{29} \mathrm{H}_{22} \mathrm{BrNO}+\mathrm{H}\right)^{+}$requires $\mathrm{m} / z$ 480.0958, found $\mathrm{m} / z$ 480.0945; ee: 82\%, AD-H, hexane isopropanol $=70 / 30, \mathrm{t}_{\mathrm{R}}=5.820$ (major), $\mathrm{t}_{\mathrm{R}}=7.357$ (minor).

(E)-3-(3-(3-chlorophenyl)-4-phenylchroman-2-yl)-1H-indole (6ka):

Yield: $72 \%$ (31.3 mg); 76:24 dr; white solid; m.p. 93.0-95.0 ${ }^{\circ} \mathrm{C}$; $[\alpha]_{\mathrm{D}}{ }^{20}=-174.8(\mathrm{c} 0.29$, acetone); ${ }^{1} \mathrm{H} \mathrm{NMR}\left(400 \mathrm{MHz} \mathrm{CDCl}_{3}\right) \delta 7.89(\mathrm{~s}, 1 \mathrm{H}), 7.77(\mathrm{~d}, J=7.8 \mathrm{~Hz}, 1 \mathrm{H}), 7.25-7.19$ (m, 2H), 7.19-7.16 (m, 3H), 7.15-7.08 (m, 2H), 7.07-7.01 (m, 2H), 6.99-6.95 (m, 1H), 6.92-6.82 $(\mathrm{m}, 3 \mathrm{H}), 6.78-6.70(\mathrm{~m}, 2 \mathrm{H}), 6.57(\mathrm{~s}, 1 \mathrm{H}), 6.44(\mathrm{~d}, J=7.6 \mathrm{~Hz}, 1 \mathrm{H}), 5.86(\mathrm{~d}, J=10.7 \mathrm{~Hz}, 1 \mathrm{H})$, $4.38(\mathrm{~d}, J=5.2 \mathrm{~Hz}, 1 \mathrm{H}), 4.17-4.08(\mathrm{~m}, 1 \mathrm{H}) ;{ }^{13} \mathrm{C}$ NMR $\left(100 \mathrm{MHz}, \mathrm{CDCl}_{3}\right) \delta 154.8,141.7,141.1$, 136.3, 133.2, 130.6, 130.3, 129.2, 128.7, 128.3, 127.6, 127.3, 126.7, 126.5, 125.9, 124.3, 123.7, $122.3,120.6,119.9,119.8,116.9,114.3,111.3,71.0,48.5,48.1$; IR (KBr): 3648, 3628, 3566, 3030, $1868,1748,1507,1456,749,701 \mathrm{~cm}^{-1} ;\left(\mathrm{C}_{29} \mathrm{H}_{22} \mathrm{ClNO}+\mathrm{H}\right)^{+}$requires $\mathrm{m} / \mathrm{z} 436.1463$, found $m / z$ 436.1462; ee: $80 \%$, AD-H, hexane/isopropanol $=70 / 30, t_{\mathrm{R}}=5.337($ minor $), \mathrm{t}_{\mathrm{R}}=6.787$ (major).

\section{(E)-3-(3-(4-chlorophenyl)-4-phenylchroman-2-yl)-1H-indole (6ma):}

Yield: 70\% (30.5 mg); 78:22 dr; white solid; m.p. 97.0-99.0 ${ }^{\circ} \mathrm{C}$; $[\alpha]_{\mathrm{D}}{ }^{20}=-106.1$ (c 0.41 , acetone); ${ }^{1} \mathrm{H} \mathrm{NMR}\left(400 \mathrm{MHz}, \mathrm{CDCl}_{3}\right) \delta 7.89$ (s, 1H), $7.76(\mathrm{~d}, J=7.8 \mathrm{~Hz}, 1 \mathrm{H}), 7.26-7.19$ $(\mathrm{m}, 2 \mathrm{H}), 7.18-7.15(\mathrm{~m}, 3 \mathrm{H}), 7.15-7.07(\mathrm{~m}, 2 \mathrm{H}), 7.07-7.00(\mathrm{~m}, 2 \mathrm{H}), 6.93-6.86(\mathrm{~m}, 4 \mathrm{H}), 6.74$ $(\mathrm{d}, J=6.9 \mathrm{~Hz}, 2 \mathrm{H}), 6.49(\mathrm{~d}, J=8.1 \mathrm{~Hz}, 2 \mathrm{H}), 5.86(\mathrm{~d}, J=10.7 \mathrm{~Hz}, 1 \mathrm{H}), 4.37(\mathrm{~d}, J=5.2 \mathrm{~Hz}$, 1H), 4.18-4.11 (m, 1H); ${ }^{13} \mathrm{C}$ NMR $\left(100 \mathrm{MHz} \mathrm{CDCl}_{3}\right) \delta 154.8,141.2,138.1,136.3,132.0$, $130.6,130.4,130.3,128.3,127.6,126.7,125.9,124.5,123.7,122.3,120.5,119.9,119.7,116.9$, 114.4, 111.3, 71.1, 48.5, 47.7; IR (KBr): 3648, 3628, 3618, 1868, 1716, 1698, 1507, 1456, 748, 
$702 \mathrm{~cm}^{-1}$; $\left(\mathrm{C}_{29} \mathrm{H}_{22} \mathrm{ClNO}+\mathrm{H}\right)^{+}$requires $\mathrm{m} / z$ 436.1463, found $\mathrm{m} / z$ 436.1449; ee: $83 \%$, IA, hexane/isopropanol $=70 / 30, \mathrm{t}_{\mathrm{R}}=5.587$ (minor), $\mathrm{t}_{\mathrm{R}}=6.463$ (major).

(E)-3-(7-methyl-3,4-diphenylchroman-2-yl)-1H-indole (6ab):

Yield: $73 \%$ (30.5 mg); 81:19 dr; white solid; m.p. $107.0-109.0{ }^{\circ} \mathrm{C} ;[\alpha]_{\mathrm{D}}{ }^{20}=-167.9(\mathrm{c} 0.37$, acetone); ${ }^{1} \mathrm{H} \mathrm{NMR}\left(400 \mathrm{MHz}, \mathrm{CDCl}_{3}\right) \delta 7.80(\mathrm{~s}, 1 \mathrm{H}), 7.78(\mathrm{~s}, 1 \mathrm{H}), 7.23-7.19(\mathrm{~m}, 1 \mathrm{H}), 7.18-7.08$ $(\mathrm{m}, 5 \mathrm{H}), 7.06-7.02(\mathrm{~m}, 1 \mathrm{H}), 7.00-6.92(\mathrm{~m}, 4 \mathrm{H}), 6.86-6.82(\mathrm{~m}, 2 \mathrm{H}), 6.74(\mathrm{~d}, J=7.5 \mathrm{~Hz}, 2 \mathrm{H}), 6.58$ $(\mathrm{d}, J=7.4 \mathrm{~Hz}, 2 \mathrm{H}), 5.91(\mathrm{~d}, J=10.7 \mathrm{~Hz}, 1 \mathrm{H}), 4.35(\mathrm{~d}, J=5.2 \mathrm{~Hz}, 1 \mathrm{H}), 4.18-4.10(\mathrm{~m}, 1 \mathrm{H}), 2.24(\mathrm{~s}$, $3 \mathrm{H}) ;{ }^{13} \mathrm{C}$ NMR $\left(100 \mathrm{MHz}, \mathrm{CDCl}_{3}\right) \delta 152.7,141.6,139.7,136.2,130.6,130.4,129.5,129.1,128.9$, 127.4, 126.4, 126.2, 126.1, 124.4, 123.7, 122.1, 119.9, 119.7, 116.5, 114.8, 111.2, 71.1, 48.8, 48.3, 20.5; IR (KBr): $3648,3586,3566,1868,1716,1496,1456,1218,740,699 \mathrm{~cm}^{-1} ;\left(\mathrm{C}_{30} \mathrm{H}_{25} \mathrm{NO}+\mathrm{H}\right)^{+}$ requires $m / z 416.2009$, found $m / z$ 416.1999; ee: 76\%, IA, hexane/isopropanol $=70 / 30$, $\mathrm{t}_{\mathrm{R}}=6.360$ (minor), $\mathrm{t}_{\mathrm{R}}=9.837$ (major).

(E)-3-(7-bromo-3,4-diphenylchroman-2-yl)-1H-indole (6ac):

Yield: 57\% (27.1 mg); 68:32 dr;

Major diastereoisomer: white solid; m.p. 81.7-82.0 ${ }^{\circ} \mathrm{C} ;[\alpha]_{\mathrm{D}}{ }^{20}=-62.5$ (c 0.20 , acetone); ${ }^{1} \mathrm{H}$ NMR (400 MHz, CDCl $) \delta 8.04(\mathrm{~s}, 1 \mathrm{H}), 7.75(\mathrm{~d}, J=7.9 \mathrm{~Hz}, 1 \mathrm{H}), 7.30(\mathrm{~d}, J=7.6 \mathrm{~Hz}, 1 \mathrm{H}), 7.23$ $(\mathrm{d}, J=7.7 \mathrm{~Hz}, 1 \mathrm{H}), 7.20-7.11(\mathrm{~m}, 3 \mathrm{H}), 7.07-6.93(\mathrm{~m}, 7 \mathrm{H}), 6.93-6.87(\mathrm{~m}, 1 \mathrm{H}), 6.73(\mathrm{~d}, J=7.3 \mathrm{~Hz}$, $2 \mathrm{H}), 6.58(\mathrm{~d}, J=7.3 \mathrm{~Hz}, 2 \mathrm{H}), 5.90(\mathrm{~d}, J=10.9 \mathrm{~Hz}, 1 \mathrm{H}), 4.40(\mathrm{~d}, J=5.1 \mathrm{~Hz}, 1 \mathrm{H}), 4.18-4.09(\mathrm{~m}$, $1 \mathrm{H}) ;{ }^{13} \mathrm{C}$ NMR $\left(100 \mathrm{MHz}, \mathrm{CDCl}_{3}\right) \delta$ 155.6, 140.8, 139.2, 136.2, 131.4, 130.5, 129.0, 127.6, 126.6, 126.4, 126.0, 123.9, 123.7, 123.5, 122.3, 121.0, 119.9, 119.8, 119.7, 114.3, 111.3, 71.7, 48.2, 47.8; IR (KBr): 3647, 2922, 2852, 1732, 1716, 1479, 1456, 1261, 741, $700 \mathrm{~cm}^{-1} ;\left(\mathrm{C}_{29} \mathrm{H}_{22} \mathrm{BrNO}+\mathrm{H}\right)^{+}$ requires $m / z$ 480.0958, found $m / z$ 480.0935; ee: 73\%, AD-H, hexane/isopropanol $=70 / 30$, $\mathrm{t}_{\mathrm{R}}=5.520$ (major), $\mathrm{t}_{\mathrm{R}}=6.530$ (minor).

Minor diastereoisomer: white solid; m.p. 85.5-87.0 ${ }^{\circ} \mathrm{C} ;[\alpha]_{\mathrm{D}}{ }^{20}=-3.0$ (c 0.17 , acetone); ${ }^{1} \mathrm{H}$ $\operatorname{NMR}\left(400 \mathrm{MHz}, \mathrm{CDCl}_{3}\right) \delta 7.84(\mathrm{~s}, 1 \mathrm{H}), 7.68(\mathrm{~d}, J=8.5 \mathrm{~Hz}, 1 \mathrm{H}), 7.40(\mathrm{~s}, 1 \mathrm{H}), 7.23-7.09$ (m, $5 \mathrm{H}), 7.04-6.93(\mathrm{~m}, 6 \mathrm{H}), 6.89-6.75(\mathrm{~m}, 5 \mathrm{H}), 5.60(\mathrm{~d}, J=10.6 \mathrm{~Hz}, 1 \mathrm{H}), 4.50(\mathrm{~d}, J=11.1 \mathrm{~Hz}, 1 \mathrm{H})$, 3.71-3.64 (m, 1H); ${ }^{13} \mathrm{C}$ NMR (100 MHz, $\left.\mathrm{CDCl}_{3}\right) \delta 155.3,143.2,140.4,136.9,130.3,129.1$, $128.3,128.2,128.1,127.7,126.6,126.5,126.4,124.8,123.8,123.2,121.3,120.8,116.9,115.7$, 114.8, 114.1, 76.8, 53.1, 50.9; IR (KBr): 3647, 2922, 1575, 1456, 1261, 1214, 1012, 798, 742, $700 \mathrm{~cm}^{-1} ;\left(\mathrm{C}_{29} \mathrm{H}_{22} \mathrm{BrNO}+\mathrm{H}\right)^{+}$requires $\mathrm{m} / \mathrm{z} 480.0958$, found $\mathrm{m} / \mathrm{z}$ 480.0931; ee: $60 \%$, OD-H, hexane isopropanol $=70 / 30, \mathrm{t}_{\mathrm{R}}=6.127$ (minor), $\mathrm{t}_{\mathrm{R}}=7.847$ (major).

(E)-3-(4-(4-fluorophenyl)-3-phenylchroman-2-yl)-1H-indole (6ad):

Yield: 59\% (24.6 mg); 75:25 dr; white solid; m.p. $105.0-107.0{ }^{\circ} \mathrm{C} ;[\alpha]_{\mathrm{D}}{ }^{20}=-135.0$ (c 0.24 , acetone); ${ }^{1} \mathrm{H}$ NMR (400 MHz, $\left.\mathrm{CDCl}_{3}\right) \delta 7.87(\mathrm{~s}, 1 \mathrm{H}), 7.78(\mathrm{~d}, J=7.7 \mathrm{~Hz}, 1 \mathrm{H}), 7.26-7.21$ $(\mathrm{m}, 2 \mathrm{H}), 7.18-7.08(\mathrm{~m}, 2 \mathrm{H}), 7.05(\mathrm{~d}, J=8.2 \mathrm{~Hz}, 1 \mathrm{H}), 7.03-6.93(\mathrm{~m}, 4 \mathrm{H}), 6.92-6.86(\mathrm{~m}, 2 \mathrm{H})$, 6.85-6.77 (m, 2H), 6.69-6.63 (m, 2H), $6.61(\mathrm{~d}, J=7.2 \mathrm{~Hz}, 2 \mathrm{H}), 5.87(\mathrm{~d}, J=10.6 \mathrm{~Hz}, 1 \mathrm{H}), 4.38$ $(\mathrm{d}, J=5.2 \mathrm{~Hz}, 1 \mathrm{H}), 4.18-4.10(\mathrm{~m}, 1 \mathrm{H}) ;{ }^{13} \mathrm{C} \mathrm{NMR}\left(100 \mathrm{MHz}, \mathrm{CDCl}_{3}\right) \delta 161.6(J=243.6 \mathrm{~Hz})$, $154.8,139.5,137.3(J=3.1 \mathrm{~Hz}), 136.2,131.9(J=7.9 \mathrm{~Hz}), 130.1,129.1,128.3,127.6,126.4,126.1$, 124.6, 123.7, 123.1, 122.2, 120.5, $119.8(\mathrm{~J}=7.7 \mathrm{~Hz}), 116.9,114.6,114.3(\mathrm{~J}=21.0 \mathrm{~Hz}), 111.2,71.1$, 48.0, 47.9; ${ }^{19} \mathrm{~F}$ NMR $\left(376 \mathrm{MHz}_{\mathrm{CDCl}}\right) \delta$-116.50; IR (KBr): 3648, 3628, 3618, 1771, 1716, 1653, $1488,1456,748,699 \mathrm{~cm}^{-1} ;\left(\mathrm{C}_{29} \mathrm{H}_{22} \mathrm{FNO}+\mathrm{H}\right)^{+}$requires $\mathrm{m} / z 420.1758$, found $\mathrm{m} / \mathrm{z} 420.1760$; ee: $76 \%$, AD-H, hexane/isopropanol $=70 / 30, \mathrm{t}_{\mathrm{R}}=5.360$ (minor), $\mathrm{t}_{\mathrm{R}}=6.863$ (major).

(E)-3-(4-(3-methoxyphenyl)-3-phenylchroman-2-yl)-1H-indole (6ae):

Yield: $80 \%$ (34.6 mg); 75:25 dr; white solid; m.p. 92.0-94.0 ${ }^{\circ} \mathrm{C} ;[\alpha]_{\mathrm{D}}{ }^{20}=-170.3(\mathrm{c} 0.27$, acetone); ${ }^{1} \mathrm{H} \mathrm{NMR}\left(400 \mathrm{MHz}, \mathrm{CDCl}_{3}\right) \delta 7.85$ (s, $\left.1 \mathrm{H}\right), 7.79(\mathrm{~d}, J=7.7 \mathrm{~Hz}, 1 \mathrm{H}), 7.25-7.20(\mathrm{~m}$, 2H), 7.16-7.08 (m, 2H), 7.07-7.05 (m, 1H), 7.05-7.01 (m, 2H), 7.01-6.94 (m, 3H), 6.92-6.86 (m, 2H), 6.74-6.68 (m, 1H), $6.64(\mathrm{~d}, J=7.3 \mathrm{~Hz}, 2 \mathrm{H}), 6.38(\mathrm{~d}, J=7.6 \mathrm{~Hz}, 1 \mathrm{H}), 6.15(\mathrm{~s}, 1 \mathrm{H}), 5.94(\mathrm{~d}$, $J=10.6 \mathrm{~Hz}, 1 \mathrm{H}), 4.37(\mathrm{~d}, J=5.2 \mathrm{~Hz}, 1 \mathrm{H}), 4.19-4.11(\mathrm{~m}, 1 \mathrm{H}), 3.54(\mathrm{~s}, 3 \mathrm{H}) ;{ }^{13} \mathrm{C} \mathrm{NMR}(100 \mathrm{MHz}$, $\left.\mathrm{CDCl}_{3}\right) \delta 158.7,154.8,143.1,139.7,136.2,130.2,129.1,128.4,128.2,127.5,126.2,126.1,124.6$, 123.7, 123.1, 122.2, 120.4, 119.9, 119.8, 116.8, 116.1, 114.7, 112.4, 111.2, 71.2, 55.0, 48.7, 48.0; IR (KBr): 3750, 3648, 3618, 3566, 1583, 1486, 1456, 1241, 744, $702 \mathrm{~cm}^{-1} ;\left(\mathrm{C}_{30} \mathrm{H}_{25} \mathrm{NO}_{2}+\mathrm{H}\right)^{+}$ 
requires $m / z$ 432.1958, found $m / z$ 432.1949; ee: 79\%, IA, hexane/isopropanol $=70 / 30$, $\mathrm{t}_{\mathrm{R}}=5.743$ (minor). $\mathrm{t}_{\mathrm{R}}=6.993$ (major).

\section{(E)-3-(6-methoxy-4-(2-methoxyphenyl)-3-phenylchroman-2-yl)-1H-indole (6af):}

Yield: $71 \%$ (32.7 mg); 90:10 dr; white solid; m.p. $110.0-112.0{ }^{\circ} \mathrm{C} ;[\alpha]_{\mathrm{D}}{ }^{20}=-133.8(\mathrm{c} 0.24$, acetone); ${ }^{1} \mathrm{H} \mathrm{NMR}\left(400 \mathrm{MHz}, \mathrm{CDCl}_{3}\right) \delta 7.81(\mathrm{~d}, J=7.6 \mathrm{~Hz}, 1 \mathrm{H}), 7.78(\mathrm{~s}, 1 \mathrm{H}), 7.21-7.13(\mathrm{~m}$, $2 \mathrm{H}), 7.14-7.02(\mathrm{~m}, 3 \mathrm{H}), 6.97(\mathrm{~d}, J=8.9 \mathrm{~Hz}, 1 \mathrm{H}), 6.93-6.85(\mathrm{~m}, 4 \mathrm{H}), 6.84(\mathrm{~d}, J=2.1 \mathrm{~Hz}, 1 \mathrm{H})$, 6.82-6.77 (m, 1H), 6.63-6.55 (m, 3H), $6.53(\mathrm{~d}, J=2.9 \mathrm{~Hz}, 1 \mathrm{H}), 5.86(\mathrm{~d}, J=10.5 \mathrm{~Hz}, 1 \mathrm{H}), 5.05$ $(\mathrm{d}, J=5.4 \mathrm{~Hz}, 1 \mathrm{H}), 4.15-4.06(\mathrm{~m}, 1 \mathrm{H}), 3.68(\mathrm{~s}, 3 \mathrm{H}), 3.09(\mathrm{~s}, 3 \mathrm{H}) ;{ }^{13} \mathrm{C} \mathrm{NMR}\left(100 \mathrm{MHz}, \mathrm{CDCl}_{3}\right)$ $\delta$ 157.5, 153.4, 149.5, 140.3, 136.2, 131.3, 130.6, 128.8, 127.6, 127.0, 126.2, 125.8, 125.5, 123.6, 122.0, 120.0, 119.9, 119.6, 117.3, 115.0, 114.7, 114.2, 111.1, 109.7, 71.2, 55.7, 54.6, 47.8, 40.0; IR (KBr): 3648, 3628, 3618, 1868, 1456, 1435, 1238, 1031, 749, $698 \mathrm{~cm}^{-1} ;\left(\mathrm{C}_{31} \mathrm{H}_{27} \mathrm{NO}_{3}+\mathrm{H}\right)^{+}$ requires $m / z$ 462.2064, found $m / z$ 462.2069; ee: 81\%, IA, hexane/isopropanol $=70 / 30$, $\mathrm{t}_{\mathrm{R}}=5.823$ (major), $\mathrm{t}_{\mathrm{R}}=7.830$ (minor).

\section{References}

1. Kochanowska-Karamyan, A.J.; Hamann, M.T. Marine Indole Alkaloids: Potential New Drug Leads for the Control of Depression and Anxiety. Chem. Rev. 2010, 110, 4489-4497. [CrossRef] [PubMed]

2. Wan, Y.; Li, Y.; Yan, C.; Yan, M.; Tang, Z. Indole: A Privileged Scaffold for the Design of Anti-Cancer Agents. Eur. J. Med. Chem. 2019, 183, 111691. [CrossRef]

3. Liu, Y.; Wu, G.; Yang, Z.; Rouh, H.; Katakam, N.; Ahmed, S.; Unruh, D.; Cui, Z.; Lischka, H.; Li, G. Multi-Layer 3D Chirality: New Synthesis, AIE and Computational Studies. Sci. China. Chem. 2020, 63, 692-698. [CrossRef]

4. Wu, G.; Liu, Y.; Yang, Z.; Jiang, T.; Katakam, N.; Rouh, H.; Ma, L.; Tang, Y.; Ahmed, S.; Rahman, A.U.; et al. Enantioselective Assembly of Multi-Layer 3D Chirality. Natl. Sci. Rev. 2020, 7, 588-599. [CrossRef] [PubMed]

5. Zhang, J.; Kürti, L. Multi-Layer 3D Chirality: Its Enantioselective Synthesis and Aggregation-Induced Emission (AIE). Natl. Sci. Rev. 2021, 8, nwaa205. [CrossRef] [PubMed]

6. Tu, M.-S.; Chen, K.-W.; Wu, P.; Zhang, Y.-C.; Liu, X.-Q.; Shi, F. Advances in Organocatalytic Asymmetric Reactions of Vinylindoles: Powerful Access to Enantioenriched Indole Derivatives. Org. Chem. Front. 2021, 8, 2643-2672. [CrossRef]

7. Sheng, F.-T.; Wang, J.-Y.; Tan, W.; Zhang, Y.-C.; Shi, F. Progresses in Organocatalytic Asymmetric Dearomatization Reactions of Indole Derivatives. Org. Chem. Front. 2020, 7, 3967-3998. [CrossRef]

8. Gioia, C.; Hauville, A.; Bernardi, L.; Fini, F.; Ricci, A. Organocatalytic Asymmetric Diels-Alder Reactions of 3-Vinylindoles. Angew. Chem. Int. Ed. 2008, 47, 9236-9239. [CrossRef]

9. Tan, B.; Hernaandez-Torres, G.; Barbas, C.F. Highly Efficient Hydrogen-Bonding Catalysis of the Diels-Alder Reaction of 3Vinylindoles and Methyleneindolinones Provides Carbazolespirooxindole Skeletons. J. Am. Chem. Soc. 2011, $133,12354-12357$. [CrossRef] [PubMed]

10. Enders, D.; Joie, C.; Deckers, K. Organocatalytic Asymmetric Synthesis of Tetracyclic Pyridocarbazole Derivatives by Using a Diels-Alder/aza-Michael/Aldol Condensation Domino Reaction. Chem. Eur. J. 2013, 19, 10818-10821. [CrossRef] [PubMed]

11. Zhang, L.-L.; Zhang, J.-W.; Xiang, S.-H.; Guo, Z.; Tan, B. Remote Control of Axial Chirality: Synthesis of Spirooxindole-Urazoles via Desymmetrization of ATAD. Org. Lett. 2018, 20, 6022-6026. [CrossRef]

12. Yang, X.; Zhou, Y.-H.; Yang, H.; Wang, S.-S.; Ouyang, Q.; Luo, Q.-L.; Guo, Q.-X. Asymmetric Diels-Alder Reaction of 3-Vinylindoles and Nitroolefins Promoted by Multiple Hydrogen Bonds. Org. Lett. 2019, 21, 1161-1164. [CrossRef]

13. Bergonzini, G.; Gramigna, L.; Mazzanti, A.; Fochi, M.; Bernardi, L.; Ricci, A. Organocatalytic Asymmetric Povarov Reactions with 2- and 3-Vinylindoles. Chem. Commun. 2010, 46, 327-329. [CrossRef]

14. Koay, W.L.; Mei, G.-J.; Lu, Y. Facile Access to Benzofuran-Fused Tetrahydropyridines via Catalytic Asymmetric [4 + 2] Cycloaddition of Aurone-Derived 1-Azadienes with 3-Vinylindoles. Org. Chem. Front. 2021, 8, 968-974. [CrossRef]

15. Chen, T.; Che, C.; Guo, Z.; Dong, X.-Q.; Wang, C.-J. Diastereoselective Synthesis of Functionalized Tetrahydropyridazines Containing Indole Scaffolds via an Inverse-Electron-Demand aza-Diels-Alder Reaction. Org. Chem. Front. 2021, 8, $4392-4398$. [CrossRef]

16. Mao, Z.; Lin, A.; Shi, Y.; Mao, H.; Li, W.; Cheng, Y.; Zhu, C. Chiral Tertiary Amine Thiourea-Catalyzed Asymmetric InverseElectron-Demand Diels-Alder Reaction of Chromone Heterodienes using 3-Vinylindoles as Dienophiles. J. Org. Chem. 2013, 78, 10233-10239. [CrossRef]

17. Guan, X.-K.; Liu, G.-F.; An, D.; Zhang, H.; Zhang, S.-Q. Chiral Imidodiphosphoric Acid-Catalyzed Highly Diastereo- and Enantioselective Synthesis of Poly-Substituted 3,4-Dihydro-2H-pyrans: [4 + 2] Cycloadditions of $\beta, \gamma$-Unsaturated $\alpha$-Ketoesters and 3-Vinylindoles. Org. Lett. 2019, 21, 5438-5442. [CrossRef] [PubMed]

18. Wu, S.-F.; Tu, M.-S.; Hang, Q.-Q.; Zhang, S.; Ding, H.; Zhang, Y.-C.; Shi, F. Construction of Chiral Chroman Scaffolds via Catalytic Asymmetric [4 + 2] Cyclizations of Paraquinone Methide Derivatives with 3-Vinylindoles. Org. Biomol. Chem. 2020, 18, 5388-5399. [CrossRef] [PubMed] 
19. Sun, X.-X.; Zhang, H.-H.; Li, G.-H.; Meng, L.; Shi, F. Diastereo- and Enantioselective Construction of an Indole-Based 2,3Dihydrobenzofuran Scaffold via Catalytic Asymmetric [3 + 2] Cyclizations of Quinone Monoimides with 3-Vinylindoles. Chem. Commun. 2016, 52, 2968-2971. [CrossRef]

20. Fan, T.; Zhang, H.-H.; Li, C.; Shen, Y.; Shi, F. The Application of N-Protected 3-Vinylindoles in Chiral Phosphoric Acid-Catalyzed [3 + 2] Cyclization with 3-Indolylmethanols: Monoactivation of the Catalyst to Vinyliminium. Adv. Synth. Catal. 2016, 358, 2017-2031. [CrossRef]

21. Zhu, Z.-Q.; Shen, Y.; Sun, X.-X.; Tao, J.-Y.; Liu, J.-X.; Shi, F. Catalytic Asymmetric [3 + 2] Cycloadditions of C-3 Unsubstituted 2-Indolylmethanols: Regio-, Diastereo- and Enantioselective Construction of the Cyclopenta[b]indole Framework. Adv. Synth. Catal. 2016, 358, 3797-3808. [CrossRef]

22. Li, C.; Xu, D.-N.; Ma, C.; Mei, G.-J.; Shi, F. Diastereo- and Enantioselective Construction of Dihydrobenzo[e]indole Scaffolds via Catalytic Asymmetric [3 + 2] Cycloannulations. J. Org. Chem. 2018, 83, 9190-9200. [CrossRef] [PubMed]

23. Mei, G.-J.; Zheng, W.; Gonçalves, T.P.; Tang, X.; Huang, K.-W.; Lu, Y. Catalytic Asymmetric Formal [3 + 2] Cycloaddition of Azoalkenes with 3-Vinylindoles: Synthesis of 2,3-Dihydropyrroles. iScience 2020, 23, 100873. [CrossRef]

24. Terada, M.; Moriya, K.; Kanomata, K.; Sorimachi, K. Chiral Brønsted Acid Catalyzed Stereoselective Addition of Azlactones to 3-Vinylindoles for Facile Access to Enantioenriched Tryptophan Derivatives. Angew. Chem. Int. Ed. 2011, 50, 12586-12590. [CrossRef]

25. Wang, Z.; Ai, F.; Wang, Z.; Zhao, W.; Zhu, G.; Lin, Z.; Sun, J. Organocatalytic Asymmetric Synthesis of 1,1-Diarylethanes by Transfer Hydrogenation. J. Am. Chem. Soc. 2015, 137, 383-389. [CrossRef] [PubMed]

26. Zhang, Y.; Zhang, S.-X.; Fu, L.-N.; Guo, Q.-X. Highly Efficient Atom-Economic Synthesis of Chiral Bis(indolyl)methanes Bearing Quaternary Stereogenic Carbon Centers. ChemCatChem 2017, 9, 3107-3110. [CrossRef]

27. Guan, X.-K.; Zhang, H.; Gao, J.-G.; Sun, D.-Y.; Qin, X.-S.; Jiang, G.-F.; Zhang, G.-L.; Zhang, S. Asymmetric Synthesis of 1,1,1Triarylethanes by Chiral Imidodiphosphoric Acid Catalyzed Nucleophilic Addition of Pyrrole and Indoles to 3-Vinylindoles. J. Org. Chem. 2019, 84, 12562-12572. [CrossRef]

28. Xue, J.-H.; Shi, M.; Yu, F.; Li, X.-Y.; Ren, W.; Fu, L.-N.; Guo, Q.-X. Catalytic Asymmetric Nucleophilic Addition of 3-Vinylindoles to Imines. Org. Lett. 2016, 18, 3874-3877. [CrossRef]

29. Li, X.-Y.; Hu, W.-T.; Xiong, Q.-J.; Ye, S.-M.; Huang, Y.-M.; Guo, Q.-X. Chiral Anion-Induced Catalytic Asymmetric Direct Dehydrative Coupling of 3-Vinylindoles and 3-Indolylmethanols. Adv. Synth. Catal. 2019, 361, 1803-1807. [CrossRef]

30. Li, J.-L.; Liu, T.-Y.; Chen, Y.-C. Aminocatalytic Asymmetric Diels-Alder Reactions via HOMO Activation. Acc. Chem. Res. 2012, 45, 1491-1500. [CrossRef]

31. Jiang, X.; Wang, R. Recent Developments in Catalytic Asymmetric Inverse-Electron-Demand Diels-Alder Reaction. Chem. Rev. 2013, 113, 5515-5546. [CrossRef] [PubMed]

32. Zhang, Y.-C.; Jiang, F.; Shi, F. Organocatalytic Asymmetric Synthesis of Indole-Based Chiral Heterocycles: Strategies, Reactions, and Outreach. Acc. Chem. Res. 2020, 53, 425-446. [CrossRef]

33. Wang, J.-Y.; Sun, M.; Yu, X.-Y.; Zhang, Y.-C.; Tan, W.; Shi, F. Atroposelective Construction of Axially Chiral Alkene-Indole Scaffolds via Catalytic Enantioselective Addition Reaction of 3-Alkynyl-2-indolylmethanols. Chin. J. Chem. 2021, 39, 2163-2171. [CrossRef]

34. Wan, X.; Sun, M.; Wang, J.-Y.; Yu, L.; Wu, Q.; Zhang, Y.-C.; Shi, F. Regio- and Enantioselective Ring-Opening Reaction of Vinylcyclopropanes with Indoles under Cooperative Catalysis. Org. Chem. Front. 2021, 8, 212-223. [CrossRef]

35. Li, T.-Z.; Liu, S.-J.; Sun, Y.-W.; Deng, S.; Tan, W.; Jiao, Y.; Zhang, Y.-C.; Shi, F. Regio- and Enantioselective [3 + 3] Cycloaddition of Nitrones with 2-Indolylmethanols Enabled by Cooperative Organocatalysis. Angew. Chem. Int. Ed. 2021, 60, $2355-2363$. [CrossRef]

36. Akiyama, T. Stronger Brønsted Acids. Chem. Rev. 2007, 107, 5744-5758. [CrossRef]

37. Terada, M. Binaphthol-derived Phosphoric Acid as a Versatile Catalyst for Enantioselective Carbon-Carbon Bond Forming Reactions. Chem. Commun. 2008, 35, 4097-4112. [CrossRef] [PubMed]

38. Terada, M. Chiral Phosphoric Acids as Versatile Catalysts for Enantioselective Transformations. Synthesis 2010, 2010, 1929-1982. [CrossRef]

39. Yu, J.; Shi, F.; Gong, L.-Z. Brønsted-Acid-Catalyzed Asymmetric Multicomponent Reactions for the Facile Synthesis of Highly Enantioenriched Structurally Diverse Nitrogenous Heterocycles. Acc. Chem. Res. 2011, 44, 1156-1171. [CrossRef]

40. Parmar, D.; Sugiono, E.; Raja, S.; Rueping, M. Complete Field Guide to Asymmetric BINOL-Phosphate Derived Brønsted Acid and Metal Catalysis: History and Classification by Mode of Activation; Brønsted Acidity, Hydrogen Bonding, Ion Pairing, and Metal Phosphates. Chem. Rev. 2014, 114, 9047-9153. [CrossRef]

41. Li, S.; Xiang, S.-H.; Tan, B. Chiral Phosphoric Acid Creates Promising Opportunities for Enantioselective Photoredox Catalysis. Chin. J. Chem. 2020, 38, 213-214. [CrossRef]

42. Liu, L.; Zhang, J. Catalytic Asymmetric [4 + 3] Cyclizations of 2-Indolylmethanols with ortho-Quinone Methides. Chin. J. Org. Chem. 2019, 39, 3308-3309. [CrossRef]

43. Tan, B. Design and Catalytic Asymmetric Construction of Axially Chiral Aryl-Alkene-Indole Frameworks. Chin. J. Org. Chem. 2020, 40, 1404-1405. [CrossRef]

44. Jiang, M.; Zhou, T.; Shi, B. Construction of a New Class of Oxindole-Based Axially Chiral Styrenes via Kinetic Resolution. Chin. J. Org. Chem. 2020, 40, 4364-4366. [CrossRef]

45. Wang, Z.; Sun, J. Recent Advances in Catalytic Asymmetric Reactions of o-Quinone Methides. Synthesis 2015, 47, 3629-3644. 
46. Caruana, L.; Fochi, M.; Bernardi, L. The Emergence of Quinone Methides in Asymmetric Organocatalysis. Molecules 2015, 20, 11733-11764. [CrossRef] [PubMed]

47. Jaworski, A.A.; Scheidt, K.A. Emerging Roles of in Situ Generated Quinone Methides in Metal-Free Catalysis. J. Org. Chem. 2016, 81, 10145-10153. [CrossRef]

48. Yang, B.; Gao, S. Recent Advances in the Application of Diels-Alder Reactions Involving o-Quinodimethanes, aza-o-Quinone Methides and $o$-Quinone Methides in Natural Product Total Synthesis. Chem. Soc. Rev. 2018, 47, 7926-7953. [CrossRef] [PubMed]

49. Lv, H.; You, L.; Ye, S. Enantioselective Synthesis of Dihydrocoumarins via N-Heterocyclic Carbene-Catalyzed Cycloaddition of Ketenes and $o$-Quinone Methides. Adv. Synth. Catal. 2009, 351, 2822-2826. [CrossRef]

50. El-Sepelgy, O.; Haseloff, S.; Alamsetti, S.K.; Schneider, C. Brønsted Acid Catalyzed, Conjugate Addition of $\beta$-Dicarbonyls to In Situ Generated ortho-Quinone Methides-Enantioselective Synthesis of 4-Aryl-4H-Chromenes. Angew. Chem. Int. Ed. 2014, 53, 7923-7927. [CrossRef]

51. Hsiao, C.-C.; Liao, H.-H.; Rueping, M. Enantio- and Diastereoselective Access to Distant Stereocenters Embedded within Tetrahydroxanthenes: Utilizing ortho-Quinone Methides as Reactive Intermediates in Asymmetric Bronsted Acid Catalysis. Angew. Chem. Int. Ed. 2014, 53, 13258-13263. [CrossRef]

52. Zhao, J.-J.; Sun, S.-B.; He, S.-H.; Wu, Q.; Shi, F. Catalytic Asymmetric Inverse-Electron-Demand oxa-Diels-Alder Reaction of In Situ Generated ortho-Quinone Methides with 3-Methyl-2-Vinylindoles. Angew. Chem. Int. Ed. 2015, 54, 5460-5464. [CrossRef] [PubMed]

53. Saha, S.; Schneider, C. Bronsted Acid-Catalyzed, Highly Enantioselective Addition of Enamides to In Situ-Generated orthoQuinone Methides: A Domino Approach to Complex Acetamidotetrahydroxanthenes. Chem. Eur. J. 2015, 21, $2348-2352$. [CrossRef]

54. Saha, S.; Schneider, C. Directing Group Assisted Nucleophilic Substitution of Propargylic Alcohols via o-Quinone Methide Intermediates: Brønsted Acid Catalyzed, Highly Enantio- and Diastereoselective Synthesis of 7-Alkynyl-12a-acetamido-Substituted Benzoxanthenes. Org. Lett. 2015, 17, 648-651. [CrossRef] [PubMed]

55. Tsui, G.C.; Liu, L.; List, B. The Organocatalytic Asymmetric Prins Cyclization. Angew. Chem. Int. Ed. 2015, 54, 7703-7706. [CrossRef]

56. Alamsetti, S.K.; Spanka, M.; Schneider, C. Synergistic Rhodium/Phosphoric Acid Catalysis for the Enantioselective Addition of Oxonium Ylides to ortho-Quinone Methides. Angew. Chem. Int. Ed. 2016, 55, 2392-2396. [CrossRef]

57. Chen, P.; Wang, K.; Guo, W.; Liu, X.; Liu, Y.; Li, C. Enantioselective Reactions of 2-Sulfonylalkyl Phenols with Allenic Esters: Dynamic Kinetic Resolution and [4+2] Cycloaddition Involving ortho-Quinone Methide Intermediates. Angew. Chem. Int. Ed. 2017, 56, 3689-3693. [CrossRef]

58. Wang, Z.; Sun, J. Enantioselective [4 + 2] Cycloaddition of $o$-Quinone Methides and Vinyl Sulfides: Indirect Access to Generally Substituted Chiral Chromanes. Org. Lett. 2017, 19, 2334-2337. [CrossRef]

59. Wang, Z.; Wang, T.; Yao, W.; Lu, Y. Phosphine-Catalyzed Enantioselective [4 + 2] Annulation of $o$-Quinone Methides with Allene Ketones. Org. Lett. 2017, 19, 4126-4129. [CrossRef]

60. Zhang, J.; Liu, X.; Guo, S.; He, C.; Xiao, W.; Lin, L.; Feng, X. Enantioselective Formal [4 + 2] Annulation of ortho-Quinone Methides with ortho-Hydroxyphenyl $\alpha, \beta$-Unsaturated Compounds. J. Org. Chem. 2018, 83, 10175-10185. [CrossRef] [PubMed]

61. Zhang, J.; Lin, L.; He, C.; Xiong, Q.; Liu, X.; Feng, X. A Chiral Scandium-Complex-Catalyzed Asymmetric Inverse-ElectronDemand oxa-Diels-Alder Reaction of $o$-Quinone Methides with Fulvenes. Chem. Commun. 2018, 54, 74-77. [CrossRef] [PubMed]

62. Spanka, M.; Schneider, C. Phosphoric Acid Catalyzed Aldehyde Addition to in Situ Generated o-Quinone Methides: An Enantioand Diastereoselective Entry toward cis-3,4-Diaryl Dihydrocoumarins. Org. Lett. 2018, 20, 4769-4772. [CrossRef]

63. Göricke, F.; Schneider, C. Palladium-Catalyzed Enantioselective Addition of Chiral Metal Enolates to In Situ Generated orthoQuinone Methides. Angew. Chem. Int. Ed. 2018, 57, 14736-14741. [CrossRef] [PubMed]

64. Jiao, P.; Nakashima, D.; Yamamoto, H. Enantioselective 1,3-Dipolar Cycloaddition of Nitrones with Ethyl Vinyl Ether: The Difference between Brønsted and Lewis Acid Catalysis. Angew. Chem. Int. Ed. 2008, 47, 2411-2413. [CrossRef]

65. Čorić, I.; List, B. Asymmetric spiroacetalization catalysed by confined Brønsted acids. Nature 2012, 483, 315-319. [CrossRef] [PubMed]

66. Greindl, J.; Hioe, J.; Sorgenfrei, N.; Morana, F.; Gschwind, R.M. Brønsted Acid Catalysis Structural Preferences and Mobility in Imine/Phosphoric Acid Complexes. J. Am. Chem. Soc. 2016, 138, 15965-15971. [CrossRef]

67. Lokesh, N.; Hioe, J.; Gramüller, J.; Gschwind, R.M. Relaxation Dispersion NMR to Reveal Fast Dynamics in Brønsted Acid Catalysis: Influence of Sterics and H-Bond Strength on Conformations and Substrate Hopping. J. Am. Chem. Soc. 2019, 141, 16398-16407. [CrossRef]

68. Luan, Y.; Schaus, S.E. Enantioselective Addition of Boronates to o-Quinone Methides Catalyzed by Chiral Biphenols. J. Am. Chem. Soc. 2012, 134, 19965-19968. [CrossRef] 